\title{
Scalar dark matter explanation of the DAMPE data in the minimal left-right symmetric model
}

\author{
Junjie Cao, ${ }^{1,2, \star}$ Xiaofei Guo, ${ }^{1,}$ Liangliang Shang, ${ }^{1, \|}$ Fei Wang, ${ }^{1,3, *}$ Peiwen Wu, ${ }^{4, \dagger}$ and Lei $\mathrm{Zu}^{5,6, \uparrow}$ \\ ${ }^{1}$ College of Physics and Materials Science, Henan Normal University, Xinxiang 453007, China \\ ${ }^{2}$ Center for High Energy Physics, Peking University, Beijing 100871, China \\ ${ }^{3}$ School of Physics, Zhengzhou University, 450000 ZhengZhou, People's Republic of China \\ ${ }^{4}$ School of Physics, KIAS, 85 Hoegiro, Seoul 02455, Republic of Korea \\ ${ }^{5}$ Key Laboratory of Dark Matter and Space Astronomy, Purple Mountain Observatory, \\ Chinese Academy of Sciences, Nanjing 210008, China \\ ${ }^{6}$ School of Astronomy and Space Science, University of Science and Technology of China, \\ Hefei 230026, Anhui, China
}

(Received 23 January 2018; revised manuscript received 6 March 2018; published 30 March 2018)

\begin{abstract}
The left-right symmetric model (LRSM) is an attractive extension of the Standard Model (SM) that can address the origin of parity violation in the SM electroweak interactions, generate tiny neutrino masses, accommodate dark matter (DM) candidates, and provide a natural framework for baryogenesis through leptogenesis. In this work, we utilize the minimal LRSM to study the recently reported DAMPE results of the cosmic $e^{+} e^{-}$spectrum, which exhibits a tentative peak around $1.4 \mathrm{TeV}$, while satisfying the current neutrino data. We propose to explain the DAMPE peak with a complex scalar DM $\chi$ in two scenarios: (1) $\chi \chi^{*} \rightarrow H_{1}^{++} H_{1}^{--} \rightarrow \ell_{i}^{+} \ell_{i}^{+} \ell_{j}^{-} \ell_{j}^{-}$, and (2) $\chi \chi^{*} \rightarrow H_{k}^{++} H_{k}^{--} \rightarrow \ell_{i}^{+} \ell_{i}^{+} \ell_{j}^{-} \ell_{j}^{-}$accompanied by $\chi \chi^{*} \rightarrow$ $H_{1}^{+} H_{1}^{-} \rightarrow \ell_{i}^{+} \nu_{\ell_{i}} \ell_{j}^{-} \nu_{\ell_{j}}$, with $\ell_{i, j}=e, \mu, \tau$ and $k=1,2$. We fit the theoretical prediction of the $e^{+} e^{-}$ spectrum to relevant experimental data to determine the scalar mass spectrum favored by the DAMPE excess. We also consider various constraints from theoretical principles and collider experiments, as well as DM relic density and direct search experiments. We find that there is ample parameter space to interpret the DAMPE data while also passing the constraints. On the other hand, our explanations usually imply the existence of other new physics at an energy scale ranging from $10^{7}$ to $10^{11} \mathrm{GeV}$. Collider tests of our explanations are also discussed.
\end{abstract}

DOI: 10.1103/PhysRevD.97.063016

\section{INTRODUCTION}

The discovery of the Higgs boson at the LHC indicates that the Standard Model (SM) of particle physics is a highly successful theory in describing a large amount of lowenergy phenomena [1,2]. On the other hand, the origin of the chiral structure of the SM-which is crucial to understanding why the matter content of the SM is much lighter than the Planck scale-is not explained in the framework of the SM. In fact, it is still unknown why the weak interaction

\footnotetext{
* Corresponding author. feiwang@zzu.edu.cn

Corresponding author. pwwu@kias.re.kr

*junjiec@itp.ac.cn

\$guoxf@gs.zzu.edu.cn

"shlwell1988@foxmail.com

"zulei@pmo.ac.cn
}

Published by the American Physical Society under the terms of the Creative Commons Attribution 4.0 International license. Further distribution of this work must maintain attribution to the author(s) and the published article's title, journal citation, and DOI. Funded by SCOAP. violates parity while all other interactions conserve parity, and whether parity conservation can be achieved at a more fundamental level.

The left-right symmetric model (LRSM), which is a vector extension of the SM with an enlarged gauge group $S U(3)_{C} \times$ $S U(2)_{L} \times S U(2)_{R} \times U(1)_{B-L}$ [3-7], assumes that the fundamental weak interaction is invariant under parity symmetry and the observed parity violation is the consequence of the spontaneous breaking of parity symmetry. Requiring the existence of right-handed neutrinos in the LRSM, tiny neutrino masses can naturally be generated by the type II seesaw mechanism [8-12]. Besides, the LRSM can accommodate dark matter candidates [13-21] and provide a natural framework for baryogenesis through leptogenesis [22]. Its gauge group can naturally appear in the typical $S O(10)$ grand unified theory (GUT) group breaking chain $[S O(10) \rightarrow$ $\left.S U(4)_{P S} \times S U(2)_{L} \times S U(2)_{R} \rightarrow L R\right]$, or from the breaking of some other partial unification theories, such as $S U(4)_{P S} \times S U(4)_{W}, S U(7)$, etc. [23-25]. The $S U(2)_{R} \times$ $U(1)_{B-L}$ breaking scale, which can be characterized by the $W_{R}$ gauge boson masses, is well motivated to lie as low as several TeV [26-32] and allows us to search for possibilities 
using various experimental signatures, such as collider signals [28,33-47], flavor observables [36,48-59], and EW precision parameters [60,61].

Very recently, the DArk Matter Particle Explorer (DAMPE) experiment reported new results of the total cosmic $e^{+}+e^{-}$flux measurement between $25 \mathrm{GeV}$ and 4.6 TeV, which contain a spectral softening at around $0.9 \mathrm{TeV}$ and a peak at around $1.4 \mathrm{TeV}[62,63]$. The spectral softening may be due to the breakdown of the conventional assumption of a continuous source distribution or the maximum acceleration limits of electron sources, while the peak can be explained by dark matter (DM) annihilation in a nearby clump halo either into an exclusive $e^{+} e^{-}$final state or equally into $e^{+} e^{-}, \mu^{+} \mu^{-}$, and $\tau^{+} \tau^{-}$states [64]. The best-fit values for the DM particle mass, annihilation cross section, DM halo mass, and DM annihilation luminosity $\mathcal{L}=\int \rho^{2} d V$ are about $1.5 \mathrm{TeV},\langle\sigma v\rangle \sim 10^{-26} \mathrm{~cm}^{3} / \mathrm{s}$, $10^{7-8} M_{\text {sun }}$, and $10^{64-66} \mathrm{GeV}^{2} \mathrm{~cm}^{-3}$, respectively, if the halo is about 0.1-0.3 kpc away from the Earth [64].

Many simplified DM models have already been proposed to interpret the DAMPE peak [65-97]. Some models are based on the typical new-gauge anomaly-free $U(1)$ family symmetry with the corresponding gauge boson as the mediator [65-71]. Other proposals, such as a scalar mediator with typical lepton-specific Yukawa couplings, have also been discussed [83-89]. However, many existing DM explanations of the DAMPE results are rather ad hoc and the involved interactions are not naturally the consequence of a well-motivated popular beyond-the-SM (BSM) model. So it is desirable to see if some popular BSM models can already explain the DAMPE results.

The minimal LRSM predicts 14 physical Higgs bosons: four $C P$-even $H_{1,2,3,4}$, two $C P$-odd $A_{1,2}$, four singly charged $H_{1,2}^{ \pm}$, and four doubly charged $H_{1,2}^{ \pm \pm}$, all with increasing masses in ascending order. In this work we propose to explain the DAMPE excess with a complex scalar DM annihilation into triplet scalar pairs which later decay and produce cosmic leptons. More specifically, we will consider the following two scenarios.

(i) Scenario I: $\chi \chi^{*} \rightarrow H_{1}^{++} H_{1}^{--} \rightarrow \ell_{i}^{+} \ell_{i}^{+} \ell_{j}^{-} \ell_{j}^{-}$.

(ii) Scenario II: $\chi \chi^{*} \rightarrow H_{k}^{++} H_{k}^{--} \rightarrow \ell_{i}^{+} \ell_{i}^{+} \ell_{j}^{-} \ell_{j}^{-}$, accompanied by $\chi \chi^{*} \rightarrow H_{1}^{+} H_{1}^{-} \rightarrow \ell_{i}^{+} \nu_{\ell_{i}} \ell_{j}^{-} \nu_{\ell_{j}}$.

Here $\chi$ stands for the scalar DM candidate, $\ell_{i, j}=e, \mu, \tau$, and $k=1,2$.

As for the proposal, we stress that the mediating scalars $H_{1,2}^{ \pm \pm}$and $H_{1}^{ \pm}$can naturally arise from the LRSM where they belong to the $S U(2)_{L}$ triplet $\Delta_{L}$ and/or the $S U(2)_{R}$ triplet $\Delta_{R}$, which are essential in generating neutrino masses. Assuming that the Dirac mass terms for neutrinos take certain forms, the Yukawa couplings involving the triplet scalars can be nearly generation universal for leptons, or the first generation can dominate over the other generations. As a result, the scalars can decay democratically into three generation of leptons or dominantly into electrons.
We also stress that DM candidates can automatically appear in LRSM with the DM stability guaranteed by either the nature of minimal dark matter or due to matter parity [13]. In the former case, the DM particle can be identified as the neutral component within certain high-dimensional $S U$ (2) representations that forbid the renormalizable couplings, leading to its decay. In the latter case, however, the residue $Z_{2}^{B-L}$ symmetry from the $U(1)_{B-L}$ breaking by the scalar triplet Higgs $\Delta_{L, R}$ can also act as the DM parity, which could guarantee the stability of alternative fermionic (bosonic) DM candidates with even (odd) $B-L$ charge $[13,14]$.

This paper is organized as follows. In Sec. II, we give a brief review of the essential features of the minimal LRSM. In Sec. III, we propose a simplified scalar DM theory based on the LRSM to explain the DAMPE excess. In Sec. IV, we discuss the implications of our explanation and its possible collider tests. Finally, we draw our conclusions in Sec. V.

\section{BRIEF REVIEW OF THE MINIMAL LRSM}

As noted previously, the LRSM model is an extension of the $\mathrm{SM}$ with the corresponding gauge group $S U(3)_{C} \times$ $S U(2)_{L} \times S U(2)_{R} \times U(1)_{B-L}$, and all of the right-handed fermions are embedded into the $S U(2)_{R}$ doublets. Due to such an assignment, right-handed neutrinos-which are needed to fit into right-handed lepton doublets—naturally appear in LRSM.

In the minimal LRSM, the quantum numbers of the particle contents under $S U(3)_{c} \times S U(2)_{L} \times S U(2)_{R} \times$ $U(1)_{B-L}$ are as follows [98].

Fermions:

$$
\begin{aligned}
Q_{L} & =\left(\begin{array}{c}
u_{L} \\
d_{L}
\end{array}\right) \in(\mathbf{3}, \mathbf{2}, \mathbf{1}, 1 / 3), \\
Q_{R} & =\left(\begin{array}{c}
u_{R} \\
d_{R}
\end{array}\right) \in(\mathbf{3}, \mathbf{1}, \mathbf{2}, 1 / 3), \\
L_{L} & =\left(\begin{array}{c}
\nu_{L} \\
\ell_{L}
\end{array}\right) \in(\mathbf{1}, \mathbf{2}, \mathbf{1},-1), \\
L_{R} & =\left(\begin{array}{c}
\nu_{R} \\
\ell_{R}
\end{array}\right) \in(\mathbf{1}, \mathbf{1}, \mathbf{2},-1) .
\end{aligned}
$$

Scalars:

$$
\begin{gathered}
\Phi=\left(\begin{array}{ll}
\phi_{1}^{0} & \phi_{1}^{+} \\
\phi_{2}^{-} & \phi_{2}^{0}
\end{array}\right) \in(\mathbf{1}, \mathbf{2}, \mathbf{2}, 0), \\
\Delta_{L}=\left(\begin{array}{cc}
\frac{\delta_{L}^{+}}{\sqrt{2}} & \delta_{L}^{++} \\
\delta_{L}^{0} & -\frac{\delta_{L}^{+}}{\sqrt{2}}
\end{array}\right) \in(\mathbf{1}, \mathbf{3}, \mathbf{1}, 2), \\
\Delta_{R}=\left(\begin{array}{cc}
\frac{\delta_{R}^{+}}{\sqrt{2}} & \delta_{R}^{++} \\
\delta_{R}^{0} & -\frac{\delta_{R}^{+}}{\sqrt{2}}
\end{array}\right) \in(\mathbf{1}, \mathbf{1}, \mathbf{3}, 2) .
\end{gathered}
$$


Here the bidoublet Higgs field is needed to give masses to ordinary SM fermions other than neutrinos, and the triplet fields are needed to generate tiny neutrino masses via mixed type-I and type-II seesaw mechanisms and while preserving the left-right symmetry.

The Lagrangian is [98]

$$
\mathcal{L}=\mathcal{L}_{\text {kin }}+\mathcal{L}_{Y}^{\Phi}+\mathcal{L}_{Y}^{\Delta}+\mathcal{L}_{L R},
$$

where the Yukawa couplings involving the bidoublets and triplet scalars are given by

$$
\begin{aligned}
-\mathcal{L}_{Y}^{\Phi}= & \overline{Q_{L}}\left(Y_{Q_{1}} \Phi+Y_{Q_{2}} \tilde{\Phi}\right) Q_{R}+\overline{L_{L}}\left(Y_{L_{1}} \Phi+Y_{L_{2}} \tilde{\Phi}\right) L_{R} \\
& + \text { H.c., } \\
-\mathcal{L}_{Y}^{\Delta}= & \overline{L_{L}^{C}} Y_{\Delta_{L}}\left(i \sigma_{2}\right) \Delta_{L} L_{L}+\overline{L_{R}^{C}} Y_{\Delta_{R}}\left(i \sigma_{2}\right) \Delta_{R} L_{R}+\text { H.c., }
\end{aligned}
$$

with $\tilde{\Phi} \equiv-\sigma_{2} \Phi^{*} \sigma_{2}$ and $\overline{\Psi^{C}}=i \Psi^{T} \gamma_{2} \gamma_{0}$, and the Higgs potential takes the form

$$
\begin{aligned}
\mathcal{L}_{L R}= & -\mu_{1}^{2} \operatorname{Tr}\left(\Phi^{\dagger} \Phi\right)-\mu_{2}^{2}\left[\operatorname{Tr}\left(\tilde{\Phi} \Phi^{\dagger}\right)+\operatorname{Tr}\left(\tilde{\Phi}^{\dagger} \Phi\right)\right]-\mu_{3}^{2}\left[\operatorname{Tr}\left(\Delta_{L} \Delta_{L}^{\dagger}\right)+\operatorname{Tr}\left(\Delta_{R} \Delta_{R}^{\dagger}\right)\right]+\lambda_{1}\left[\operatorname{Tr}\left(\Phi^{\dagger} \Phi\right)\right]^{2}+\lambda_{2}\left\{\left[\operatorname{Tr}\left(\tilde{\Phi} \Phi^{\dagger}\right)\right]^{2}\right. \\
& \left.+\left[\operatorname{Tr}\left(\tilde{\Phi}^{\dagger} \Phi\right)\right]^{2}\right\}+\lambda_{3} \operatorname{Tr}\left(\tilde{\Phi} \Phi^{\dagger}\right) \operatorname{Tr}\left(\tilde{\Phi}^{\dagger} \Phi\right)+\lambda_{4} \operatorname{Tr}\left(\Phi^{\dagger} \Phi\right)\left[\operatorname{Tr}\left(\tilde{\Phi} \Phi^{\dagger}\right)+\operatorname{Tr}\left(\tilde{\Phi}^{\dagger} \Phi\right)\right]+\rho_{1}\left\{\left[\operatorname{Tr}\left(\Delta_{L} \Delta_{L}^{\dagger}\right)\right]^{2}+\left[\operatorname{Tr}\left(\Delta_{R} \Delta_{R}^{\dagger}\right)\right]^{2}\right\} \\
& +\rho_{2}\left[\operatorname{Tr}\left(\Delta_{L} \Delta_{L}\right) \operatorname{Tr}\left(\Delta_{L}^{\dagger} \Delta_{L}^{\dagger}\right)+\operatorname{Tr}\left(\Delta_{R} \Delta_{R}\right) \operatorname{Tr}\left(\Delta_{R}^{\dagger} \Delta_{R}^{\dagger}\right)\right]+\rho_{3} \operatorname{Tr}\left(\Delta_{L} \Delta_{L}^{\dagger}\right) \operatorname{Tr}\left(\Delta_{R} \Delta_{R}^{\dagger}\right)+\rho_{4}\left[\operatorname{Tr}\left(\Delta_{L} \Delta_{L}\right) \operatorname{Tr}\left(\Delta_{R}^{\dagger} \Delta_{R}^{\dagger}\right)\right. \\
& \left.+\operatorname{Tr}\left(\Delta_{L}^{\dagger} \Delta_{L}^{\dagger}\right) \operatorname{Tr}\left(\Delta_{R} \Delta_{R}\right)\right]+\alpha_{1} \operatorname{Tr}\left(\Phi^{\dagger} \Phi\right)\left[\operatorname{Tr}\left(\Delta_{L} \Delta_{L}^{\dagger}\right)+\operatorname{Tr}\left(\Delta_{R} \Delta_{R}^{\dagger}\right)\right]+\left\{\alpha _ { 2 } e ^ { i \delta _ { 2 } } \left[\operatorname{Tr}\left(\tilde{\Phi} \Phi^{\dagger}\right) \operatorname{Tr}\left(\Delta_{L} \Delta_{L}^{\dagger}\right)\right.\right. \\
& \left.\left.+\operatorname{Tr}\left(\tilde{\Phi}^{\dagger} \Phi\right) \operatorname{Tr}\left(\Delta_{R} \Delta_{R}^{\dagger}\right)\right]+ \text { H.c. }\right\}+\alpha_{3}\left[\operatorname{Tr}\left(\Phi \Phi^{\dagger} \Delta_{L} \Delta_{L}^{\dagger}\right)+\operatorname{Tr}\left(\Phi^{\dagger} \Phi \Delta_{R} \Delta_{R}^{\dagger}\right)\right]+\beta_{1}\left[\operatorname{Tr}\left(\Phi \Delta_{R} \Phi^{\dagger} \Delta_{L}^{\dagger}\right)+\operatorname{Tr}\left(\Phi^{\dagger} \Delta_{L} \Phi \Delta_{R}^{\dagger}\right)\right] \\
& +\beta_{2}\left[\operatorname{Tr}\left(\tilde{\Phi} \Delta_{R} \Phi^{\dagger} \Delta_{L}^{\dagger}\right)+\operatorname{Tr}\left(\tilde{\Phi}^{\dagger} \Delta_{L} \Phi \Delta_{R}^{\dagger}\right)\right]+\beta_{3}\left[\operatorname{Tr}\left(\Phi \Delta_{R} \tilde{\Phi}^{\dagger} \Delta_{L}^{\dagger}\right)+\operatorname{Tr}\left(\Phi^{\dagger} \Delta_{L} \tilde{\Phi} \Delta_{R}^{\dagger}\right)\right] .
\end{aligned}
$$

In the above potential, $\mu_{i}, \lambda_{i}, \beta_{i}$ (with $i=1,2,3$ ) and $\rho_{j}, \alpha_{j}$ (with $j=1, \ldots, 4$ ) are all free parameters.

The $S U(2)_{R} \times U(1)_{B-L}$ is broken to $U(1)_{Y}$ by the vacuum expectation value (VEV) of the $S U(2)_{R}$ triplet scalar $\Delta_{R}$, while the SM gauge group is broken to $U(1)_{Q}$ by the VEVs of the bidoublet Higgs. The VEVs of the bidoublet and triplets, which are taken to be real to forbid spontaneous $C P$ violation, are parametrized as

$$
\begin{aligned}
& \left\langle\phi_{1}^{0}\right\rangle=\frac{v}{\sqrt{2}} \cos \beta, \quad\left\langle\phi_{2}^{0}\right\rangle=\frac{v}{\sqrt{2}} \sin \beta, \quad t_{\beta} \equiv \tan \beta=\frac{v_{2}}{v_{1}}, \\
& \left\langle\delta_{L}^{0}\right\rangle=\frac{v_{L}}{\sqrt{2}}, \quad\left\langle\delta_{R}^{0}\right\rangle=\frac{v_{R}}{\sqrt{2}},
\end{aligned}
$$

with $v_{L} \ll v \ll v_{R}$, so $v$ can be identified as the SM VEV. Therefore, the masses of the new gauge bosons read

$$
M_{Z_{R}} \simeq \sqrt{g_{R}^{2}+g_{B L}^{2}} v_{R}, \quad M_{W_{R}} \simeq \frac{g_{R}}{\sqrt{2}} v_{R} .
$$

Due to the LR symmetry, we take the two $S U(2)$ gauge couplings to be equal, namely, $g_{R}=g_{L}$. The mixing between the electric charged gauge fields $W_{L}$ and $W_{R}$ will result in two mass eigenstates $W$ and $W^{\prime}$, and similarly the mixing among the neutral components $W_{L}^{3}, W_{R}^{3}$, and $B_{B-L}$ will predict three vector bosons as mass eigenstates, i.e., the photon, $Z$ and $Z^{\prime}$.

The minimal LRSM predicts ten physical particles: four $C P$-even Higgs bosons, two $C P$-odd Higgs bosons, two singly charged Higgs bosons, and two doubly charged Higgs bosons. With the minimization conditions of the scalar potential, one can trade the parameters $\mu_{i}$ and $\beta_{2}$ using the VEV [98]. As a result, with the assumption that $v_{L} / v, v_{L} / v_{R}, \tan \beta, \alpha_{1}, \alpha_{2}, \beta_{1} \rightarrow 0$ (so that the mixings between the bidoublet and the triplet scalars are small), the bidoublet-like scalar masses are given by

$m_{h}^{2} \simeq 2 \lambda_{1} v^{2}-\frac{8 \lambda_{4}^{2} v^{4}}{\alpha_{3} v_{R}^{2}}, \quad m_{H}^{2} \simeq 2\left(2 \lambda_{2}+\lambda_{3}\right) v^{2}+\frac{\alpha_{3}}{2} v_{R}^{2}$,

$$
m_{A}^{2} \simeq \frac{\alpha_{3}}{2} v_{R}^{2}+2\left(\lambda_{3}-2 \lambda_{2}\right) v^{2}, \quad m_{H^{ \pm}}^{2} \simeq \frac{1}{4} \alpha_{3}\left(v^{2}+2 v_{R}^{2}\right),
$$

where $h$ corresponds to the SM-like Higgs boson with its mass fixed at $125 \mathrm{GeV}$, and $H, A$, and $H^{ \pm}$are the heavier neutral scalar and pseudoscalar states and the charged Higgs, respectively. The triplet-scalar sector masses are

$$
\begin{aligned}
m_{H_{L}}^{2} \simeq \frac{1}{2}\left(\rho_{3}-2 \rho_{1}\right) v_{R}^{2}, \quad m_{H_{R}}^{2} \simeq 2 \rho_{1} v_{R}^{2}, \\
m_{A_{L}}^{2} \simeq \frac{1}{2}\left(\rho_{3}-2 \rho_{1}\right) v_{R}^{2}, \\
m_{H_{L}^{ \pm}}^{2} \simeq \frac{1}{2}\left(\rho_{3}-2 \rho_{1}\right) v_{R}^{2}, \\
m_{H_{a}^{ \pm \pm}}^{2} \simeq 2 \rho_{2} v_{R}^{2}+\frac{1}{2} \alpha_{3} v^{2}, \\
m_{H_{b}^{ \pm \pm}}^{2} \simeq \frac{1}{2}\left(\left(\rho_{3}-2 \rho_{1}\right) v_{R}^{2}+\alpha_{3} v^{2}\right),
\end{aligned}
$$


where particles with an index $L(R)$ mostly consist of $\Delta_{L(R)}$ components, and since the doubly charged Higgses can in general be strongly mixed, we label them as $H_{a / b}^{ \pm \pm}$. Under the condition $v_{L} / v_{R} \rightarrow 0$, the mass spectrums implies the approximate degeneracies

$$
\begin{gathered}
2 \lambda_{1} v^{2}-\frac{8 \lambda_{4}^{2} v^{4}}{\alpha_{3} v_{R}^{2}} \simeq m_{h}^{2}, \\
\frac{\alpha_{3}}{2} v_{R}^{2} \simeq m_{H}^{2} \simeq m_{A}^{2} \simeq m_{H^{ \pm}}^{2}
\end{gathered}
$$

for the bidoublet sector and

$$
\begin{gathered}
2 \rho_{1} v_{R}^{2} \simeq m_{H_{R}}^{2}, \\
2 \rho_{2} v_{R}^{2}+\frac{1}{2} \alpha_{3} v^{2} \simeq m_{H_{a}^{ \pm \pm}}^{2}, \\
\frac{\rho_{3}-2 \rho_{1}}{2} v_{R}^{2} \simeq m_{H_{b}^{ \pm \pm}}^{2} \simeq m_{H_{L}^{ \pm}}^{2} \simeq m_{H_{L}}^{2} \simeq m_{A_{L}}^{2}
\end{gathered}
$$

for the triplet sector. In the following, we label particles with the same quantum numbers using the subscripts $1,2, \ldots$. and assume that they have an ascending mass order, i.e.,

$$
\begin{gathered}
m_{H_{1,2,3,4}}: \text { neutral } C P \text {-even Higgs, } \\
m_{A_{1,2}}: \text { neutral } C P \text {-odd Higgs, } \\
m_{H_{1,2}^{ \pm}}: \text {singly charged Higgs, } \\
m_{H_{1,2}^{ \pm \pm}}: \text {doubly charged Higgs. }
\end{gathered}
$$

In the minimal LRSM, the tiny neutrino mass can be generated via mixed type-I and type-II seesaw mechanisms with the corresponding mass matrix given by [98]

$$
\frac{1}{2}\left(\begin{array}{ll}
\overline{\nu_{L}} & \overline{\nu_{R}^{C}}
\end{array}\right)\left(\begin{array}{ll}
M_{L}^{*} & M_{D} \\
M_{D}^{T} & M_{R}
\end{array}\right)\left(\begin{array}{c}
\nu_{L}^{C} \\
\nu_{R}
\end{array}\right)+\text { H.c. },
$$

where

$$
\begin{aligned}
M_{L} & =\sqrt{2} Y_{\Delta_{L}} v_{L}, \quad M_{R}=\sqrt{2} Y_{\Delta_{R}} v_{R}, \\
\text { and } \quad M_{D} & =\frac{v}{\sqrt{2}}\left(Y_{L_{1}} \sin \beta+Y_{L_{2}} \cos \beta\right) .
\end{aligned}
$$

After the diagonalization of the mass matrix, the Majorana mass of the left-handed neutrinos can be determined to be

$$
m_{\nu}^{\text {light }}=\left(M_{L}^{*}-M_{D} M_{R}^{-1} M_{D}^{T}\right) .
$$

This expression indicates that a possibly large cancellation among the two terms is required to give tiny neutrino masses of order $0.1 \mathrm{eV}$. One should note that loop corrections will in general spoil the cancellation among the two terms.
The discrete LR symmetry, which can be identified with parity symmetry, requires the Yukawa couplings to satisfy [98]

$$
Y_{a}=Y_{a}^{\dagger}, \quad Y_{\Delta_{L}}=Y_{\Delta_{R}} .
$$

In terms of the neutrino masses and Pontecorvo-MakiNakagawa-Sakata mixing matrix from neutrino oscillation experiments, the Yukawa couplings involving the triplets can be determined as [98]

$$
\begin{aligned}
Y_{\Delta}^{( \pm \pm \pm)} \equiv & Y_{\Delta_{L / R}}^{( \pm \pm \pm)}=\frac{1}{2 \sqrt{2} v_{L}} M_{D}^{* 1 / 2} R^{*} \operatorname{diag}\left(B_{D}^{(i, i)}\right. \\
& \pm \sqrt{\left.\left(B_{D}^{(i, i)}\right)^{2}+4 \alpha\right)} R^{\dagger} M_{D}^{1 / 2} \\
B_{D}= & R^{\dagger} M_{D}^{*-1 / 2} m_{\nu}^{\text {light }} M_{D}^{-1 / 2} R^{*} \\
\alpha= & v_{L} / v_{R}
\end{aligned}
$$

for given specific inputs of $v_{L}, v_{R}$, and $M_{D}$. In the previous expression, $B_{D}$ is a diagonal $3 \times 3$ matrix and $R$ is a unitary rotation matrix to keep both sides of Eq. (2.22) equal. It should be noted that there is not a unique solution to the triplet-Yukawa couplings, which corresponds to an ambiguous \pm sign in the bracket of the expression. It should also be noted that the magnitude of $Y_{\Delta}$ is sensitive to the choice of $v_{L}$ and $M_{D}$, and may vary from $\mathcal{O}\left(10^{-4}\right)$ to $\mathcal{O}(1)$ in producing the measured neutrino masses and mixings.

\section{DAMPE EXPLANATION WITH SCALAR DM}

As one of the most compelling BSM theories, the LRSM itself can naturally accommodate a DM candidate, which is absolutely stable due to the residual matter parity $Z_{2}^{B-L}$ $[13,14]$. To provide a scalar DM candidate, the minimal realization requires the introduction of two complex scalar fields $\phi_{L}$ and $\phi_{R}$ with their respective quantum numbers under the gauge group $S U(3)_{c} \times S U(2)_{L} \times S U(2)_{R} \times$ $U(1)_{B-L}[14]$,

$$
\begin{gathered}
\phi_{L}=\left(\begin{array}{c}
\phi_{L}^{0} \\
\phi_{L}^{-}
\end{array}\right) \in(\mathbf{1}, \mathbf{2}, \mathbf{1},-\mathbf{1}), \\
\phi_{R}=\left(\begin{array}{c}
\phi_{R}^{0} \\
\phi_{R}^{-}
\end{array}\right) \in(\mathbf{1}, \mathbf{1}, \mathbf{2},-\mathbf{1}),
\end{gathered}
$$

and the DM candidate corresponds to the lightest mass eigenstate among the mixture of the neutral components. ${ }^{1}$

\footnotetext{
${ }^{1}$ Note that for such an assignment of gauge quantum numbers, the properties of the fields $\phi_{L}$ and $\phi_{R}$ are similar to those of the left-handed and right-handed scalar lepton fields in the supersymmetric LRSM, respectively. Thus, in some cases the DM as the lightest state can mimic the behavior of the popular sneutrino $\mathrm{DM}$ in supersymmetric theories.
} 
These additional gauge nonsinglet scalars greatly extend the minimal LRSM, and the general form of the resulting theory contains 16 new parameters and 20 extra quartic scalar interactions [14]. Obviously, working in such a complex framework to interpret the DAMPE excess involves the treatment of a large number of free parameters, which usually obscures the underlying physics. This situation motivates us to consider the incorporation of DM physics into the LRSM in a simpler way. To be more specific, we note that the gauge-singlet scalar is ubiquitous in various UV-completion theories of the LRSM. For example, it can appear in the decomposition of the $\mathbf{5 4}$ or 210 dimensional representation of $S O(10)$ under the PatiSalam $S U(4)_{c} \times S U(2)_{L} \times S U(2)_{R}$ (or LR gauge group) gauge group and possibly be tuned to be light [99]. It can also appear in orbifold GUT models with proper boundary conditions by embedding the LRSM gauge group into GUT or partial unification theory $[100,101]$. These facts motivate us to consider a VEV-less gauge-singlet complex scalar particle as the DM candidate with its stability protected by an accidental global $U(1)_{\chi}$ symmetry, which may be promoted to a gauged one that was broken at a certain high energy scale into a discrete $Z_{2}$ symmetry in the early evolution of the Universe.

Based on the above discussion, we propose to introduce a complex scalar DM $\chi$ into the minimal LRSM-which is a singlet under the LR gauge group — as the simplest effective DM model to explain the DAMPE results with the DM stability guaranteed by the conserved accidental $U(1)_{\chi}$ quantum number, just as the conserved baryon number classically ensures the stability of proton. The couplings of $\chi$ are assumed to be

$$
\begin{aligned}
\mathcal{L} \supseteq & \left|\partial_{\mu} \chi\right|^{2}-\mu_{\chi}^{2}|\chi|^{2}-\kappa_{1}|\chi|^{2}\left[\operatorname{Tr}\left(\Delta_{L} \Delta_{L}^{\dagger}\right)\right. \\
& \left.+\operatorname{Tr}\left(\Delta_{R} \Delta_{R}^{\dagger}\right)\right]-\kappa_{2}|\chi|^{2} \operatorname{Tr}\left(\Phi^{\dagger} \Phi\right) \\
& -\kappa_{3}|\chi|^{2}\left[\operatorname{Tr}\left(\tilde{\Phi} \Phi^{\dagger}\right)+\operatorname{Tr}\left(\tilde{\Phi}^{\dagger} \Phi\right)\right]-\lambda_{\chi}|\chi|^{4},
\end{aligned}
$$

which are invariant under the discrete left-right symmetry for real parameters $\lambda_{\chi}$ and $\kappa_{i}(i=1,2,3)$.

We use SARAH [102] to implement the model and SPHENo $[103,104]$ to calculate the mass spectrum. Since many parameters are involved in our discussion on the interpretation of the excess, we fix some of them in Table I, where $v_{L}=2 \times 10^{-7} \mathrm{GeV}, M_{D}=\mathbb{1} \mathrm{MeV}$, and the " +++ " sign choice for three generations will give

$$
Y_{\Delta}^{(+++)}=\left(\begin{array}{ccc}
1.12 \times 10^{-2} & -1.41 \times 10^{-5} & 2.97 \times 10^{-6} \\
-1.41 \times 10^{-5} & 1.12 \times 10^{-2} & -3.78 \times 10^{-5} \\
2.97 \times 10^{-6} & -3.78 \times 10^{-5} & 1.12 \times 10^{-2}
\end{array}\right)
$$

according to Eq. (2.22). This setup-where the Dirac neutrino mass is diagonal and flavor universal-always
TABLE I. Parameter settings for the scan in this work over the varying parameters. These parameters are defined at the scale $v_{R}=20 \mathrm{TeV}$.

\begin{tabular}{lccc}
\hline \hline$v_{L}$ & $2.0 \times 10^{-7} \mathrm{GeV}$ & $v_{R}$ & $2.0 \times 10^{4} \mathrm{GeV}$ \\
$M_{D}$ & $\mathbb{1} \mathrm{MeV}$ & $\left.Y_{\Delta}^{(++}+\right)$ & Eq. $(3.3)$ \\
$m_{\chi}$ & $(2.95,3.15) \mathrm{TeV}$ & $\tan \beta$ & $10^{-4}$ \\
$\lambda_{1}$ & 0.13 & $\lambda_{2}$ & 0 \\
$\lambda_{3}$ & 0 & $\lambda_{4}$ & 0 \\
$\rho_{1}$ & $(0,0.1)$ & $\rho_{2}$ & $(0,0.1)$ \\
$\rho_{3}$ & $(0,0.2)$ & $\rho_{4}$ & 0 \\
$\alpha_{1}$ & 0 & $\alpha_{2}$ & 0 \\
$\alpha_{3}$ & 2.0 & $\beta_{1}$ & 0 \\
$\beta_{3}$ & 0 & $\kappa_{1}$ & $(0,5)$ \\
$\kappa_{2,3}$ & 0 & $\lambda_{\chi}$ & 0 \\
\hline \hline
\end{tabular}

predicts an almost degenerate spectrum of right-handed neutrinos due to the nearly degenerate diagonal $Y_{\Delta}^{(i, i)}$ entries and the relatively smaller nondiagonal entries. As a result, the triplet-dominated scalars $H_{1,2}^{ \pm \pm}$will decay dominantly into $l_{i}^{ \pm} l_{i}^{ \pm}(i=e, \mu, \tau)$ with approximately equal branching ratios.

In practice, in order to obtain the solutions to the DAMPE excess we first determine the favored DM mass $m_{\chi}$ and $\Delta m \equiv m_{\chi}-m_{H_{1}^{ \pm \pm}}$, where we utilize the process $\chi \chi \rightarrow H_{1}^{++} H_{1}^{--} \rightarrow \ell_{i}^{+} \ell_{i}^{+} \ell_{j}^{-} \ell_{j}^{-}$(with $\ell_{i, j}=e, \mu, \tau$ ) to generate the measured $e^{+} e^{-}$spectrum. The impact of the mass spectrum on the $e^{+} e^{-}$flux as well as our strategy to determine their favored region have been described in detail in Ref. [71]. Here we simply apply them to the case in which the intermediate scalars $H_{1}^{ \pm \pm}$as the DM direct annihilation products decay democratically into $e^{ \pm} e^{ \pm}$, $\mu^{ \pm} \mu^{ \pm}$, and $\tau^{ \pm} \tau^{ \pm}$. The results are presented in Fig. 1, where we show the fit of the predicted $e^{+} e^{-}$spectrum to the corresponding AMS-02 and DAMPE data. The left panel is the $\chi^{2}$ map on the $\Delta m-m_{\chi}$ plane with the color bar denoting the values of $\chi^{2}$ and the enclosed line corresponding to the constant contour $\chi^{2}=\chi_{\text {best }}^{2}+2.3$. The region bounded by this contour is interpreted as the best region of the two-step DM annihilation process to explain the DAMPE excess at the $1 \sigma$ level. The best-fit point is located at about $(6 \mathrm{GeV}, 3060 \mathrm{GeV})$ with $\langle\sigma v\rangle_{0}=2.98 \times$ $10^{-26} \mathrm{~cm}^{3} / \mathrm{s}$ for the default setting on the distance of the subhalo from the Earth and the subhalo mass in Ref. [71], $d=0.1 \mathrm{kpc}$ and $M_{\text {halo }}=1.9 \times 10^{7} m_{\odot}$. The right panel of Fig. 1 corresponds to the $e^{+} e^{-}$spectrum predicted by the best-fit point which lowers the $\chi^{2}$ value to 104.2 in comparison with 109.7 for the background-only hypothesis. These facts indicate that, by choosing appropriate $\left(\Delta m, m_{\chi}\right)$, the process $\chi \chi \rightarrow H_{1}^{++} H_{1}^{--} \rightarrow l^{+} l^{+} l^{-} l^{-}$ is indeed capable of reproducing the DAMPE $e^{+} e^{-}$peak.

Next, we discuss in detail two scenarios to explain the excess, which are presented in Table II. In order to get relevant parameter points, we scan the parameters $m_{\chi}, \kappa_{1}$, 

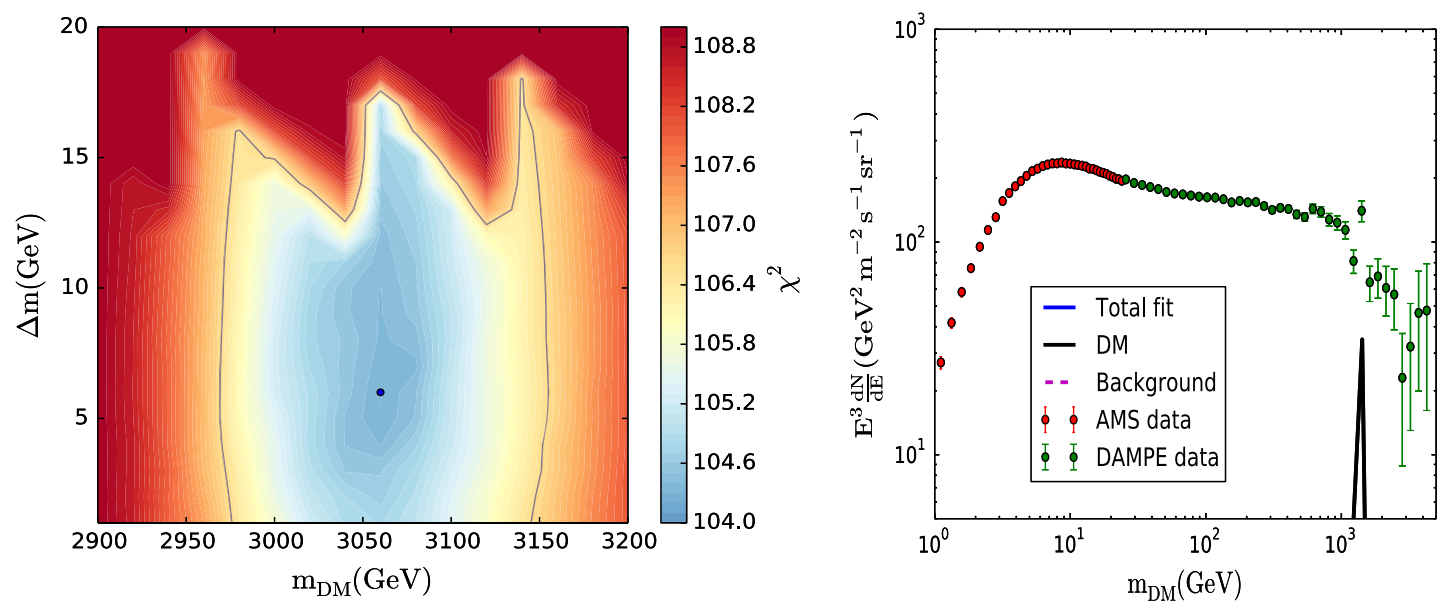

FIG. 1. Fit of the $e^{+} e^{-}$spectrum generated by the process $\chi \chi \rightarrow H_{1}^{++} H_{1}^{--}$with $H_{1}^{ \pm \pm} \rightarrow e^{ \pm} e^{ \pm}, \mu^{ \pm} \mu^{ \pm}, \tau^{ \pm} \tau^{ \pm}$at a rate equal to the AMS02 and DAMPE data. Left: $\chi^{2}$ map projected onto the $\Delta m-m_{\chi}$ plane with $\Delta m \equiv m_{\chi}-m_{H_{1}^{ \pm \pm}}$, where the color bar denotes the $\chi^{2}$ values. The best-fit point if located at about $(6 \mathrm{GeV}, 3060 \mathrm{GeV})$, and the contour of $\chi^{2}=\chi_{\text {best }}^{2}+2.3$ (solid line) is also shown. Right: The cosmic $e^{+} e^{-}$spectrum of the best-fit point generated by the DM annihilation process in comparison with the AMS-02 and DAMPE data.

and $\rho_{i}(i=1,2,3)$ with the setting in Table $\mathrm{I}$, and we consider the following constraints:

(1) The DM relic density $\Omega_{\mathrm{DM}}=0.1199 \pm 0.0027[105$, 106], which implies that $\langle\sigma v\rangle_{\mathrm{FO}} \sim \mathcal{O}\left(10^{-26}\right) \mathrm{cm}^{3} / \mathrm{s}$ with a velocity $v \sim 0.1 c$ in early freeze-out.

In our theoretical framework, the DM annihilation into scalar pairs $S S^{*}$ proceeds through the quartic scalar interaction $\chi \chi^{*} S S^{*}$, the $s$-change exchange of any $C P$-even Higgs boson, and the $t$-channel exchange of $\chi$. Therefore, the relic density mainly limits the coupling strength $\kappa_{1}$ for the parameter settings in Table I and the favored spectrum in Fig. 1. We use MICROMEGAs [107,108] to obtain the density at which the threshold effects are important when the DM mass is close to intermediate particle masses [109]. We also use MICROMEGAs to calculate the DM annihilation rate in today's Universe and the DM-nucleon scattering rate discussed below.

(2) The mass spectrum presented in Fig. 1 as well as today's DM annihilation cross section $\langle\sigma v\rangle_{0}>1 \times$ $10^{-26} \mathrm{~cm}^{3} / \mathrm{s}$ with $v \sim 10^{-3} \mathrm{c}$ in the nearby subhalo, which are essential conditions to explain the DAMPE result (see Refs. [64,71] for more details).
Obviously, the former condition limits the ranges of $m_{\chi}$ and $\rho_{i}$, while the latter condition (as a useful supplement to the DM relic density) has nontrivial requirements on $\kappa_{1}$.

(3) DM direct-detection bounds on the spin-independent DM-nucleon scattering cross section $\sigma_{\chi-n}^{\text {SI }}$ from the recent XENON-1T [110] and PandaX-II experiments [111].

In our theory, the scattering proceeds through $t$ channel exchange of any $C P$-even Higgs boson [112]. Considering that the $C P$-even Higgs fields in the bidoublet $\Phi$ have no coupling with $\chi$ since we set $\kappa_{2}=\kappa_{3}=0$ in Table I, and that the $C P$-even field in $\Delta_{R}$ which couples to $\chi \chi^{*}$ with a strength proportional to $\kappa_{1} v_{R}$ has no couplings with quarks due to the $U(1)_{B-L}$ charge assignment, one can conclude that the scattering rate vanishes if there is no mixing between these two types of fields in forming mass eigenstates, i.e., the magnitude of the rate is decided by the size of the mixing. As far as the parameter setting in Table I is concerned, we checked that the mixing is less than $10^{-8}$, which results in a scattering rate less than $10^{-14} \mathrm{pb}$. Therefore, although the

TABLE II. Two scenarios of the Higgs spectrum and DM annihilation channels pertinent to explaining the DAMPE excess.

\begin{tabular}{|c|c|c|}
\hline Scenario & $\begin{array}{l}\text { Mass spectrum: } m_{\chi} \text { and } \Delta m>0 \text { lie within } \\
\text { the region enclosed by the solid line in Fig. } 1 .\end{array}$ & $\begin{array}{l}\text { Relevant DM annihilations } \\
\ell_{i, j}=e, \mu, \tau, k=1,2\end{array}$ \\
\hline I & $\begin{array}{l}m_{H_{1}^{ \pm \pm}} \sim 3 \mathrm{TeV} \text { and } \Delta m \equiv m_{\chi}-m_{H_{\mathrm{D}}^{ \pm \pm}} \\
\text {The other scalars are heavier than DM. }\end{array}$ & $\chi \chi \rightarrow H_{1}^{++} H_{1}^{--} \rightarrow \ell_{i}^{+} \ell_{i}^{+} \ell_{j}^{-} \ell_{j}^{-}$ \\
\hline II & $\begin{array}{c}m_{\chi}>m_{H_{2}^{ \pm \pm}}, m_{H_{2}}, m_{A_{1}}, m_{H_{1}^{ \pm}}>m_{H_{1}^{ \pm \pm}} \\
\text {and } \Delta m \equiv m_{\chi}-m_{H_{1}^{ \pm \pm}} \\
\text {The other scalars are heavier than DM. }\end{array}$ & $\begin{aligned} \chi \chi & \rightarrow H_{k}^{++} H_{k}^{--} \rightarrow \ell_{i}^{+} \ell_{i}^{+} \ell_{j}^{-} \ell_{j}^{-} \\
\chi \chi & \rightarrow H_{1}^{+} H_{1}^{-} \rightarrow \ell_{i}^{+} \nu_{\ell_{i}} \ell_{j}^{-} \nu_{\ell_{j}}\end{aligned}$ \\
\hline
\end{tabular}




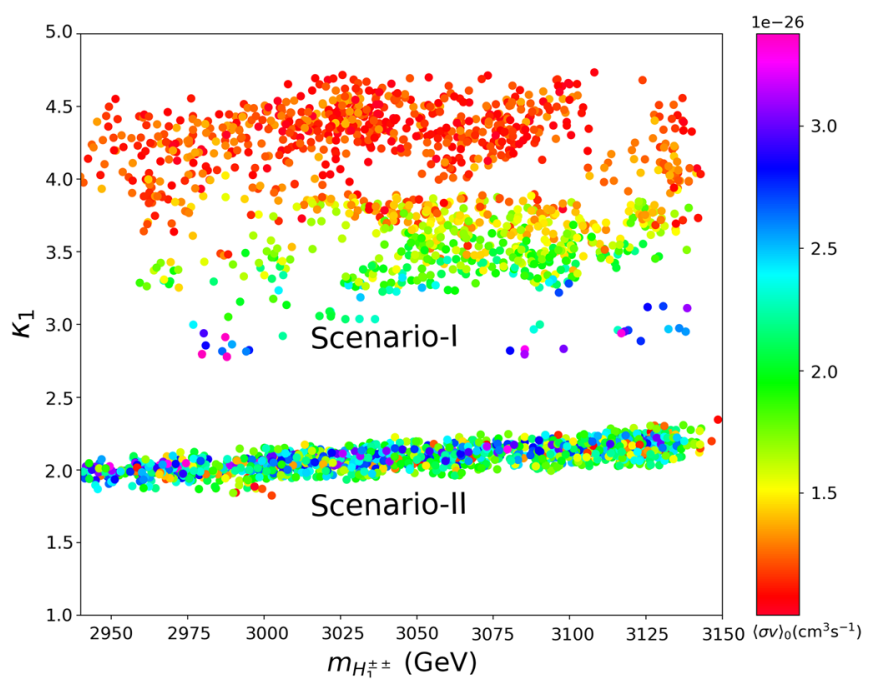

FIG. 2. Scenario-I and Scenario-II samples from Table II to explain the DAMPE data, which are projected onto the $\kappa_{1}-m_{H_{1}^{ \pm \pm}}$ plane with the color bar denoting the value of $\langle\sigma v\rangle_{0}$. The upper and lower regions correspond to Scenario I and II, respectively, and all of the samples satisfy the constraints listed in the main text.

direct-detection bounds play an important role in DM physics and must be considered when explaining the excess, they actually impose no constraint on our case.

The surviving samples from the scan are projected onto the $m_{H_{1}^{ \pm \pm}}-\kappa_{1}$ plane in Fig. 2 with the color bar indicating the $\mathrm{DM}$ annihilation rate today $\langle\sigma v\rangle_{0}$. This figure indicates that there are certain parameter regions that explain the excess without conflicting with experimental results, which are characterized by $\kappa_{1} \in(2.8,5)$ for Scenario I, $\kappa_{1} \in(1.8,2.5)$ for Scenario II, and $\langle\sigma v\rangle_{0} \in(1,3.2) \times$ $10^{-26} \mathrm{~cm}^{3} \mathrm{~s}^{-1}$ for both scenarios. In Table III we present more details about the relevant parameter regions. Note that $\kappa_{1}$ in Scenario I is significantly larger than that in Scenario II. The underlying reason is that $\kappa_{1}$ is the coupling that controls the interaction strength between DM $\chi$ and Higgs triplets, as indicated in Eq. (3.2). Because a more intermediate Higgs is available in the two-step DM annihilation in Scenario II (see Table II), a relatively low $\kappa_{1}$ is enough to predict the right relic density. Also note that $\kappa_{1}$ in Scenario I spans a much wider range than that in Scenario II. This is because in some rare cases of Scenario I, the DM may annihilate through the resonant $\mathrm{H}_{3}$ into right-handed neutrinos. We checked these cases and found that, although $H_{1}^{++} H_{1}^{--}$is still the dominant annihilation product, the contribution of the neutrino channel to the total annihilation rate may reach about $35 \%$ at the freeze-out temperature.

Regarding the DM explanation of the DAMPE excess, we emphasize that it is consistent with the other direct and indirect DM experiments (see Refs. [65,78] for a detailed discussion), such as the H.E.S.S. data on the annihilation $\chi \chi \rightarrow S^{*} S \rightarrow 4 e$ [97,113], the Fermi-LAT data in the direction of the dwarf spheroidal galaxies [114], the Planck cosmic microwave background (CMB) data which is sensitive to energy injection in the CMB from DM annihilations [115,116], and the IceCube data on DM annihilation into neutrinos [117]. Dark matter in our scenario also survives the upper bounds from the XENON-10 and XENON-100 experiments on DM-electron scattering [118]. Moreover, we checked that the samples in Fig. 2 also satisfy the constraints from collider experiments and some theoretical principles recently discussed in Refs. [30-32], which mainly limit the parameters $\rho_{i}$. These constraints include the following ${ }^{2}$

(1) The existence of a SM-like Higgs boson (corresponding to $H_{1}$ in our case) with a mass around $125 \mathrm{GeV}$ : We examine its properties with the package HigGSSignals [119].

(2) Collider searches for extra scalars: We calculate the couplings of the non-SM-like Higgs bosons using SPHENO and link them to HigGsBounds [120-122]. We require them to be allowed by the direct search results at colliders.

(3) Low-energy lepton-flavor-violation processes considered in Ref. [98], which include two-body decays such as $\mu \rightarrow e \gamma, \tau \rightarrow e \gamma, \tau \rightarrow \mu \gamma$, and three-body decays such as $\mu \rightarrow e e e, \tau \rightarrow e e e$ : These processes proceed at loop level and may be enhanced greatly (in comparison with their SM predictions) by large flavor nondiagonal elements of $Y_{\Delta}$ if the new scalars and new vector bosons running in the loops are not too heavy. In our analysis, we use FLAVORKIT [123] to calculate the rates of these processes.

(4) $B_{s, d}^{0}-\bar{B}_{s, d}^{0}$ mixing: As shown in Ref. [55], this constraint is rather strong and it requires the masses of the heavier doublet Higgses to be larger than about $20 \mathrm{TeV}$. In our discussion, we satisfy the requirement by setting $\alpha_{3}=2$ and $v_{R}=20 \mathrm{TeV}$.

(5) The precision electroweak observable $\delta \rho$, which is defined by $\delta \rho \equiv \frac{\Pi_{Z Z}(0)}{m_{Z}^{2}}-\frac{\Pi_{W W}(0)}{m_{W}^{2}}$ with $\Pi_{Z Z}(0)$ and $\Pi_{W W}(0)$ denoting the self-energy of the $Z$ and $W$ boson at zero momentum, respectively $[124,125]^{3}$ : In the minimal LRSM, the new scalars enter the selfenergies, and consequently $\delta \rho$ depends on their spectrum [60]. In our analysis we use SPHENO to calculate $\delta \rho$ and find that its typical size is less than

\footnotetext{
${ }^{2}$ Note that these limitations are obviously weak for the parameter settings in Table I. This is because the $Y_{\Delta}$ we adopt is small in magnitude and nearly flavor diagonal, all scalars other than the SM-like Higgs boson as well as the new gauge bosons are heavier than about $3 \mathrm{TeV}$ and thus their effects are decoupled, $v_{L}=2 \times 10^{-7} \mathrm{GeV}$ is tiny, and the quartic scalar couplings are only moderately large.

${ }^{3}$ Note that in our case, the tree-level contribution to the $\rho$ parameter is negligibly small since we consider a very small $v_{L}[60]$.
} 
TABLE III. Survived parameter ranges to explain the DAMPE excess. Note that the values of $\rho_{i}(i=1,2,3)$ in the table are scaled by a factor of $10^{2}$ and the DM mass is in unit of TeV.

\begin{tabular}{lcccccccc}
\hline \hline \multicolumn{9}{c}{ Scenario I } \\
\hline$m_{\chi}$ & $\kappa_{1}$ & $100 \rho_{1}$ & $100 \rho_{2}$ & $100 \rho_{3}$ & $\kappa_{1}$ & $100 \rho_{1}$ & $100 \rho_{2}$ & $100 \rho_{3}$ \\
$(2.9,3.2)$ & $(2.8,4.7)$ & $(1,5)$ & $(1.1,1.2)$ & $(8,20)$ & $(1.8,2.4)$ & $(1,3)$ & $(1.1,1.2)$ & $(6,10)$ \\
\hline \hline
\end{tabular}

$10^{-4}$, which lies within the experimentally allowed region $-0.000313 \leq \delta \rho \leq 0.00156[126,127]$.

(vi) The vacuum stability condition, $\lambda_{1} \geq 0, \rho_{1} \geq 0, \rho_{1}+$ $\rho_{2} \geq 0$ and $\rho_{1}+2 \rho_{2} \geq 0$, which was derived in Refs. [128,129].

(vii) Unitary constraints on the quartic scalar couplings: Since the theory predicts 11 complex fields and 17 independent quartic couplings, the unitary constraints are rather complicated in a general case. In our analysis, however, we note that $\alpha_{3}$ and $\kappa_{1}$ are much larger than the other couplings. So we consider a simple case where only $\alpha_{3}$ and $\kappa_{1}$ among the couplings are nonzero. We work in the basis $\left(\chi \chi^{*}\right.$, $H_{L} H_{L}, A_{L} A_{L}, H_{L}^{ \pm} H_{L}^{\mp}, H_{L}^{ \pm \pm} H_{L}^{\mp \mp}, H_{R} H_{R}, A_{R} A_{R}$, $H_{R}^{ \pm} H_{R}^{\mp}, H_{R}^{ \pm \pm} H_{R}^{\mp \mp}, H_{1} H_{1}, A_{1} A_{1}, H_{1}^{ \pm} H_{1}^{\mp}, H_{2} H_{2}$, $A_{2} A_{2}, H_{2}^{ \pm} H_{2}^{\mp}$ ), and calculate all $2 \rightarrow 2$ transition amplitudes as in Refs. [130-132]. After the diagonalization of the transition matrix, we obtain the following unitarity condition:

$$
4 \kappa_{1}^{2}+9 \alpha_{3}^{2}+\sqrt{16 \kappa_{1}^{4}+28 \kappa_{1}^{2} \alpha_{3}^{2}+15 \alpha_{3}^{4}} \leq(8 \pi)^{2} .
$$

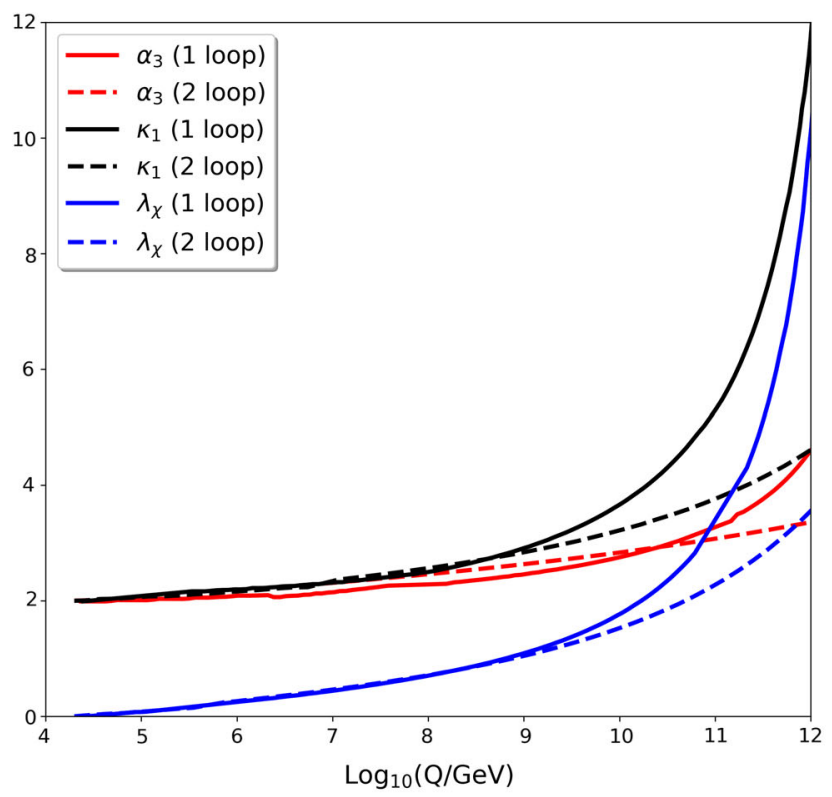

We note that the unitarity constraint was also discussed in Ref. [32], where the authors sorted out all possible quartic contact terms in terms of the physical fields where the vertex factors of each coupling are linear functions of the quartic couplings. Finally, the authors required each of the couplings to be less than $8 \pi$. Obviously, the limits obtained in this way are rather conservative, which can be seen from their results:

$$
\alpha_{1} \leq 8 \pi, \quad \alpha_{2} \leq 4 \pi, \quad \alpha_{1}+\alpha_{3} \leq 8 \pi, \ldots
$$

\section{IMPLICATION OF THE EXPLANATION}

From the discussion in Sec. III, one can learn that our explanation relies on a moderately large $\kappa_{1}$, i.e., $\kappa_{1} \sim 4$ for Scenario I and $\kappa_{1} \sim 2$ for Scenario II. This may spoil the perturbativity of the theory. We investigate this issue by first choosing one benchmark point in Scenario II and presenting its properties in Table IV. Then, we study the behaviors of some potentially large couplings with the increase of the energy scale according to the renormalization group equations (RGEs) presented in the Appendix.

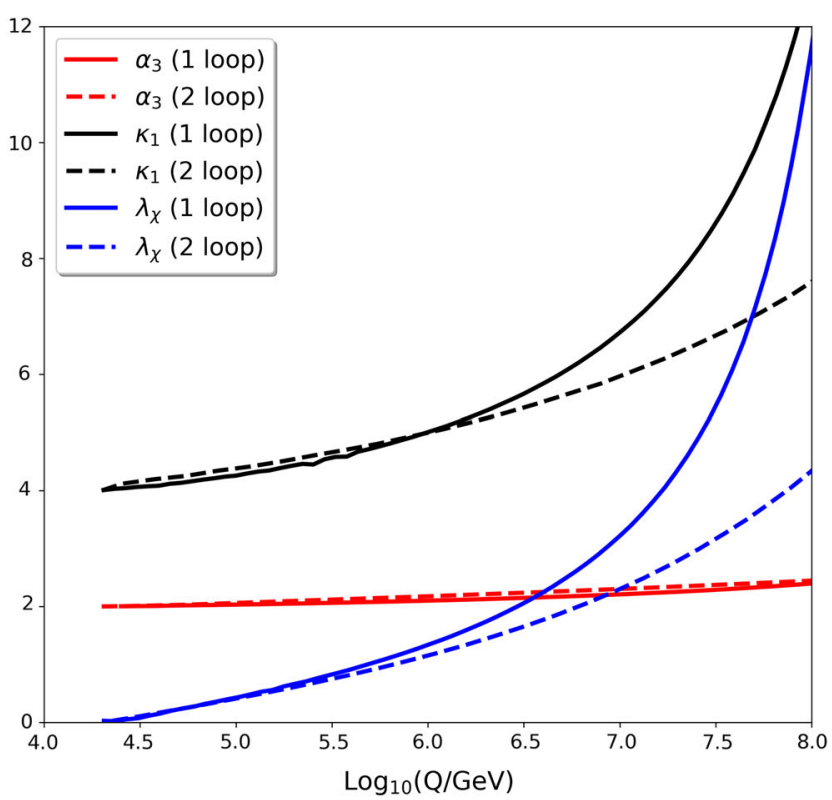

FIG. 3. Left: The RGE evolution of the three largest couplings— $-\kappa_{1}$ (black lines), $\lambda_{\chi}$ (blue lines), and $\alpha_{3}$ (red lines)—for the benchmark point of Scenario II in Table IV. In calculating the RGE, only the dominant contributions are included, and the solid and dashed lines correspond to one-loop and two-loop results, respectively. Right: Same as the left panel, but for $\kappa_{1}=4$ which is favored by Scenario I to explain the DAMPE excess. 
TABLE IV. A benchmark point in Scenario II. Here the nearly degenerate particles $H_{2}^{0}, A_{1}, H_{1}^{ \pm}$, and $H_{1}^{++}$correspond to triplet scalars, and $H_{4}^{0}, A_{2}$, and $H_{2}^{ \pm}$are bidoublet scalars.

\begin{tabular}{lccc}
\hline \hline$\kappa_{1}$ & 2.0 & $\rho_{1}$ & $1.4 \times 10^{-2}$ \\
$\rho_{2}$ & $1.1 \times 10^{-2}$ & $\rho_{3}$ & $7.3 \times 10^{-2}$ \\
$m_{H_{1}^{0}}$ & $1.25 \times 10^{2} \mathrm{GeV}$ & $m_{H_{2}^{0}}$ & $3.01 \times 10^{3} \mathrm{GeV}$ \\
$m_{H_{3}^{0}}$ & $3.34 \times 10^{3} \mathrm{GeV}$ & $m_{H_{4}^{0}}$ & $2.00 \times 10^{4} \mathrm{GeV}$ \\
$m_{A_{1}}$ & $3.01 \times 10^{3} \mathrm{GeV}$ & $m_{A_{2}}$ & $2.00 \times 10^{4} \mathrm{GeV}$ \\
$m_{H_{1}^{ \pm}}$ & $3.01 \times 10^{3} \mathrm{GeV}$ & $m_{H_{2}^{+}}$ & $2.00 \times 10^{4} \mathrm{GeV}$ \\
$m_{H_{1}^{ \pm \pm}}$ & $2.99 \times 10^{3} \mathrm{GeV}$ & $m_{H_{2}^{ \pm \pm}}$ & $3.01 \times 10^{3} \mathrm{GeV}$ \\
$m_{Z^{\prime}}$ & $1.57 \times 10^{4} \mathrm{GeV}$ & $m_{W^{\prime}}$ & $9.37 \times 10^{3} \mathrm{GeV}$ \\
$\Gamma_{Z^{\prime}}$ & $4.66 \times 10^{2} \mathrm{GeV}$ & $\Gamma_{W^{\prime}}$ & $3.27 \times 10^{2} \mathrm{GeV}$ \\
$\operatorname{Br}\left(Z^{\prime} \rightarrow H_{1,2}^{++} H_{1,2}^{--}\right)$ & $\sim 2.8 \%$ & $\operatorname{Br}\left(W^{\prime+} \rightarrow H_{1}^{+} H_{2}^{0}\right)$ & $\sim 5.1 \times 10^{-5}$ \\
$\operatorname{Br}\left(H_{1}^{++} \rightarrow l_{i}^{+} l_{i}^{+}\right)$ & $\sim 33 \%, 33 \%, 33 \%$ & $\operatorname{Br}\left(H_{2}^{++} \rightarrow l_{i}^{+} l_{i}^{+}\right)$ & $\sim 33 \%, 33 \%, 33 \%$ \\
$\operatorname{Br}\left(H_{1}^{+} \rightarrow l_{j}^{+} \nu_{i}\right)$ & $\operatorname{Br}\left(H_{2}^{0} \rightarrow \nu_{i} \nu_{i}\right)$ & $\sim 33 \%, 33 \%, 33 \%$ \\
\hline \hline
\end{tabular}

In the left panel of Fig. 3, we show the results for the benchmark point, with the solid and dashed lines denoting one-loop and two-loop predictions, respectively. This indicates that $\kappa_{1}$ as the largest coupling is the first one to reach its Landau pole at a scale near $4 \times 10^{12} \mathrm{GeV}$ at the one-loop level, while at the two-loop level the growth rate of $\kappa_{1}$ to higher energy scales is greatly reduced. In fact, for an energy scale lower than about $10^{9} \mathrm{GeV}$ (where $\kappa_{1} \simeq 3$ ), the difference between the one-loop and two-loop $\beta$ functions is negligibly small. However, for an energy scale higher than about $10^{10} \mathrm{GeV}$ (where $\kappa_{1} \simeq 4$ ), the difference between the one-loop and two-loop $\beta$ functions is significant. This fact implies that the perturbativity of the theory becomes much worse and perturbative calculations are no longer reliable near $10^{10} \mathrm{GeV}$. As a comparison, we choose $\kappa_{1}=4$ (favored by Scenario I to explain the excess) while keeping $\alpha_{3}$ and $\lambda_{\chi}$ the same, and study its RGE behavior. We find that $\kappa_{1}$ reaches its Landau pole at the scale $3.2 \times$ $10^{8} \mathrm{GeV}$ at the one-loop level, as shown in the right panel of Fig. 3. Similar to the case in the left panel, the sharp increasing behavior at the one-loop level is ameliorated after including the two-loop corrections near $10^{7} \mathrm{GeV}$. We should note that the appearance of the Landau pole may not be problematic. Possible new particles (such as heavy fermions or new gauge bosons) that can contribute to the beta function at high energy (such as the Pati-Salam unification scale) may change the RGE behavior of the quartic couplings at such an energy scale. We also recall that the calculation of the two-loop results is rather involved, and we obtain our results using PyR@TE [133-135]. To the best of our knowledge, the two-loop result in the minimal LRSM is still absent in the literature.

The discussion in Sec. III also indicates that the explanation requires one or more doubly charged Higgses $H_{1,2}^{ \pm \pm}$with masses around $3 \mathrm{TeV}$. These particles, once produced at future colliders, will decay dominantly into same-sign lepton pairs with an invariant mass peaking around $3 \mathrm{TeV}$. Given that such a signal is quite distinct at hadron colliders, we briefly discuss its observability in future experiments. In Fig. 4, we show for the benchmark point in Table IV the production rates $\sigma(p p \rightarrow$ $H_{1}^{++} H_{1}^{--}, H_{2}^{++} H_{2}^{--}$) which proceed mainly through the exchange of vector bosons such as $\gamma, Z$, and $Z^{\prime}$, as well as through neutral Higgs bosons $H_{i}$ (but with smaller contributions). This figure indicates that the production rates increase with the collision energy $\sqrt{s}$, but are generally small even for $\sqrt{s}=100 \mathrm{TeV}$ which has $\sigma \sim 0.1 \mathrm{fb}$. This implies that even if one neglects the SM background arising from possibly mistagged leptons, an integrated luminosity of $\gtrsim \mathcal{O}(100) \mathrm{fb}^{-1}$ may be needed to detect the signal.

In Fig. 4 we also show the production rates of $\sigma\left(p p \rightarrow H_{1}^{+} H_{2}^{0}, H_{1}^{-} H_{2}^{0}\right)$. Since $H_{1}^{-}$and $H_{2}^{0}$ mainly decay into $\ell \nu$ and neutrino pairs, respectively, these processes will result in a mono- $\ell+E_{\text {miss }}^{T}$ signal which has the same signature as $W Z$-associated production and thus is also distinct. Nevertheless, since the corresponding production rates are too small (at most $10^{-2} \mathrm{fb}$ ), the prospect of detecting them may be dim.

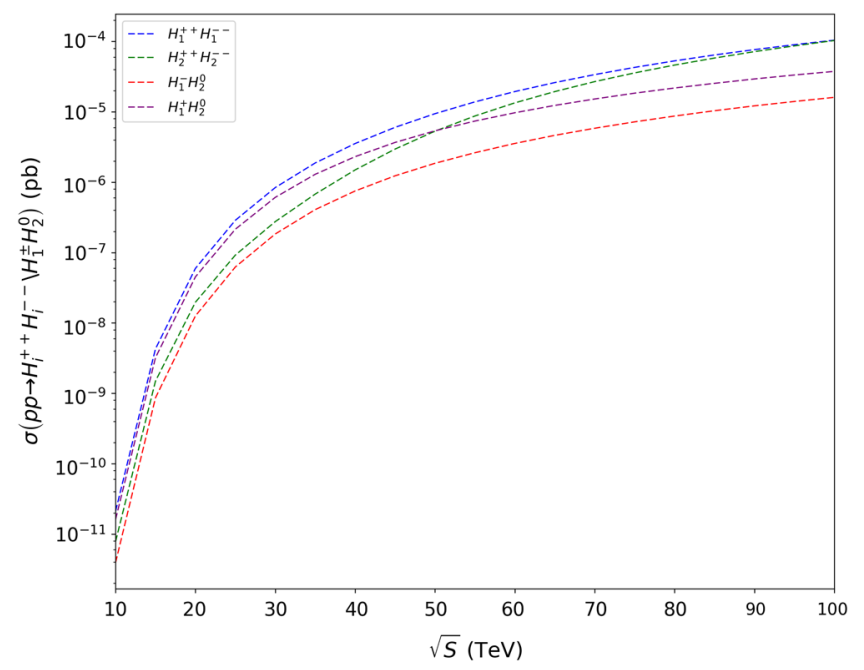

FIG. 4. Production rates of $\sigma\left(p p \rightarrow H_{1}^{++} H_{1}^{--}, H_{2}^{++} H_{2}^{--}\right.$, $\left.H_{1}^{+} H_{2}^{0}, H_{1}^{-} H_{2}^{0}\right)$ for the benchmark point of Scenario II in Table IV as a function of the collision energy $\sqrt{s}$. 
For the benchmark point in Table IV, we also checked that the production rates of $\sigma\left(p p \rightarrow H_{1}^{++} H_{2}^{--}, H_{1}^{+} A_{1}^{0}\right)$ are much smaller than those of the above production channels. Moreover, we note that the doubly charged Higgses $H_{1,2}^{ \pm \pm}$ may contribute to the process $e^{+} e^{-} \rightarrow \ell_{i}^{+} \ell_{j}^{-}$via $t$-channel mediation at the future International Linear Collider. However, the signal strength of this process is proportional to $Y_{\Delta}^{4}$. Due to the small value of $Y_{\Delta}$ as well as the heavy mass of the $H_{1,2}^{ \pm \pm}$, we estimate that the effect is negligible.

\section{CONCLUSION}

Given the fact that the electroweak interaction in the SM violates parity while all other interactions conserve parity, the LRSM is an attractive extension of the SM that can address the parity violation in the EW interaction, generate tiny neutrino masses, accommodate DM candidates, and provide a framework for baryogenesis through leptogenesis. In this work we enlarged the field content of the minimal LRSM by adding one gaugesinglet scalar field, which acts as the simplest extension of the LRSM to include DM physics, and utilized the resulting theory to study the recently reported DAMPE results of the cosmic $e^{+} e^{-}$flux. We considered two scenarios to explain the DAMPE peak with a scalar $\operatorname{DM} \chi$ : (1) $\chi \chi^{*} \rightarrow H_{1}^{++} H_{1}^{--} \rightarrow \ell_{i}^{+} \ell_{i}^{+} \ell_{j}^{-} \ell_{j}^{-}$, and (2) $\chi \chi^{*} \rightarrow$ $H_{k}^{++} H_{k}^{--} \rightarrow \ell_{i}^{+} \ell_{i}^{+} \ell_{j}^{-} \ell_{j}^{-}$accompanied by $\chi \chi^{*} \rightarrow H_{1}^{+} H_{1}^{-} \rightarrow$ $\ell_{i}^{+} \nu_{\ell_{i}} \ell_{j}^{-} \nu_{\ell_{j}}$ (with $\ell_{i, j}=e, \mu, \tau$ and $k=1,2$ ). We fit the theoretical prediction of the $e^{+} e^{-}$spectrum to relevant experimental data to determine the scalar mass spectrum favored by the DAMPE excess. We also considered various constraints from theoretical principles, collider experiments, the DM relic density, and direct search experiments. We found that there is ample parameter space that can interpret the DAMPE data while passing the constraints. Our interpretation, on the other hand, implies the breakdown of the perturbativity of the theory at an energy scale ranging from $10^{7}$ to $10^{11} \mathrm{GeV}$, which can be avoided by the intervention of other new physics. We also briefly discussed collider signals of our explanation and concluded that a luminosity of $\gtrsim \mathcal{O}(100) \mathrm{fb}^{-1}$ is needed to probe the signal at a future hadronic collider with $\sqrt{s}=100 \mathrm{TeV}$.

Before we end this work, we would like to clarify two important points.

One is that the gauge-singlet scalar DM considered in this work is motivated by some UV completions of the LRSM and also by simplicity. The two essential ingredients of our interpretation of the excess include the existence of one or more leptophilic mediators $S_{i}$ (which are $H_{1,2}^{ \pm \pm}$and $H_{1}^{ \pm}$in the LRSM), and sufficiently large quartic scalar couplings $\chi \chi^{*} S_{i} S_{i}^{*}$ which ensure that the $\mathrm{DM} \chi$ acquires the right relic density without any suppression of the $\langle\sigma v\rangle_{0}$ for the annihilation $\chi \chi^{*} \rightarrow S_{i} S_{i}^{*}$. It should be noted that the scalar leptophilic DM model containing $\phi_{L}, \phi_{R}$ introduced in the beginning of Sec. III also possesses the aforementioned ingredients, where the $\lambda_{\Delta_{1}}$ term in Eq. (64) and the $\lambda_{\phi \Delta \Delta \phi}$ term in Eq. (67) of Ref. [14] play the same role as the $\kappa_{1}$ term in DM physics. Therefore, the model may also be utilized to explain the excess; however, its structure is much more complex. Obviously, if the DM explanation of the excess is confirmed by future data, a careful examination of the model should be carried out, which is beyond the scope of this work.

We should compare this work with our earlier studies $[67,71]$, where we extended the SM using lepton-specific symmetries and built anomaly-free theories in an economic way. In all of these works we considered a scalar DM candidate to generate the $e^{+} e^{-}$flux using a two-step annihilation process, followed by solving the propagation equation of the electron/positron in a cosmic ray and looking for the maximum value of the likelihood function to attain the $\chi^{2}$ map in the $\Delta m-m_{\chi}$ plane needed to produce the right shape of the $e^{+} e^{-}$spectrum. Then, we obtained the parameter space of the model relevant to explaining the excess by considering the constraints from DM relic density and direct-detection experiments. Due to their shared workflow procedures, we organized the discussions in a similar way. However, the underlying physics in these works are quite different, which is reflected in the following aspects.

(1) The theoretical frameworks considered in Refs. [67,71] focus on the DAMPE excess, and the DM candidate together with the lepton-specific gauge bosons are added by hand. By contrast, in this work we considered the physically well-motivated LRSM where the leptophilic mediators and the DM candidates can arise quite naturally without the needs for lepton-specific gauge interactions. This model, aside from being capable of explaining the DAMPE excess, can also account for many fundamental problems in particle physics such as neutrino masses and baryogenesis. Moreover, as we mentioned in the Introduction, the theory has rich phenomenology at colliders which can be tested in the future. Consequently, it is of particular interest.

(2) In earlier works $[67,71]$ we extended the SM using certain lepton-specific gauge symmetries in an economic way. In doing this, we noted that the anomaly-free condition puts a nontrivial requirement on the quantum number of leptons for the new symmetry and, consequently, the new gauge boson $Z^{\prime}$ as the mediator of the two-step annihilation must decay in certain pattern, e.g., either democratically into three generations of lepton pairs or into $e^{+} e^{-}$ and $\mu^{+} \mu^{-}$with equal branching ratios. By contrast, in this work we considered the triplet scalars as the mediators of the DM annihilation. The decay modes of the scalars are determined by the Yukawa coupling $Y_{\Delta}$ which depends on the parameters $v_{L, R}$ and $M_{D}$ and is therefore somewhat arbitrary. Moreover, since the LRSM predicts six triplet Higgs 
bosons, we have more choices when selecting the mediators of the annihilation than in Refs. [67,71]. These features make the discussions of this work more adaptive to future cosmic-ray data.

\section{ACKNOWLEDGMENTS}

F. W. thanks the Henan Normal University for kind hospitality during his visit there. We thank J. Chakrabortty from the Indian Institute of Technology for the usage of the tool PYR@TE. This work is supported by the National Natural Science Foundation of China (NNSFC) under Grants No. 11575053 and 11675147, and also by the Innovation Talent project of Henan Province under Grant No. 15HASTIT017, by the Young Core instructor foundation of the Henan Education Department.

\section{APPENDIX: RGES OF THE RELEVANT COUPLINGS}

Here we present the RGEs of the parameters in the extended minimal LRSM discussed in this work. Since the complete forms of the equations in the LRSM are quite complicated $[32,136]{ }^{4}$ we consider the case of large $\kappa_{1}, \lambda_{\chi}$, and $\alpha_{3}$ and only include potentially large contributions in the RGEs. At the initial stage of this work, we calculated the effects of $\kappa_{1}$ and $\lambda_{\chi}$ on the one-loop $\beta$ functions of the couplings by hand, and took the rest of the contributions from Ref. [136]. While revising the manuscript, we noticed that the tool PYR@TE [133-135] can calculate the $\beta$ functions automatically. Thus, we use this program to calculate all of the one-loop $\beta$ functions. We find that, as far as the dominant contributions listed below are concerned, the two sets of results agree with each other. We also calculate the two-loop $\beta$ functions for the couplings $\kappa_{1}, \lambda_{\chi}, \alpha_{3}$, and $\alpha_{1}$ using the program and keep only the dominant terms. We note that the calculation of the two-loop contributions is rather computationally heavy, and attaining the complete set of the two-loop results is beyond the capability of our computing resources.

The resulting RGEs are given by

$$
\begin{aligned}
& 16 \pi^{2} \frac{\partial g_{s}}{\partial t}=-7 g_{s}^{3}, \quad 16 \pi^{2} \frac{\partial g_{2}}{\partial t}=-\frac{7}{3} g_{2}^{3}, \quad 16 \pi^{2} \frac{\partial g_{B L}}{\partial t}=\frac{14}{3} g_{B L}^{3}, \\
& 16 \pi^{2} \frac{\partial y_{t}}{\partial t}=y_{t}\left(-\frac{2}{3} g_{B L}^{2}-\frac{9}{2} g_{2}^{2}-8 g_{s}^{2}+8 y_{t}^{2}\right) \\
& 16 \pi^{2} \frac{\partial \kappa_{1}}{\partial t}=4 \kappa_{1}^{2}+8 \lambda_{\chi} \kappa_{1}+16 \rho_{1} \kappa_{1}+6 \rho_{3} \kappa_{1}-12 \kappa_{1} g_{2}^{2}-6 \kappa_{1} g_{B L}^{2}+\frac{\mathbf{1}}{\mathbf{1 6} \pi^{2}}\left(-\mathbf{4} \alpha_{\mathbf{1}}^{2} \kappa_{\mathbf{1}}-\mathbf{3} \alpha_{\mathbf{3}}^{2} \kappa_{\mathbf{1}}-\mathbf{1 5} \kappa_{\mathbf{1}}^{3}-\mathbf{4 8} \kappa_{\mathbf{1}}^{2} \lambda_{\chi}-\mathbf{4 0} \kappa_{\mathbf{1}} \lambda_{\chi}^{2}-\mathbf{4} \alpha_{\mathbf{1}} \alpha_{\mathbf{3}} \kappa_{\mathbf{1}}\right), \\
& 16 \pi^{2} \frac{\partial \lambda_{\chi}}{\partial t}=20 \lambda_{\chi}^{2}+6 \kappa_{1}^{2}+\frac{\mathbf{1}}{\mathbf{1 6} \pi^{2}}\left(-\mathbf{2 4} \kappa_{1}^{3}-\mathbf{6 0} \kappa_{1}^{2} \lambda_{\chi}-\mathbf{2 4 0} \lambda_{\chi}^{\mathbf{3}}\right), \\
& 16 \pi^{2} \frac{\partial \lambda_{1}}{\partial t}=32 \lambda_{1}^{2}+\frac{5}{2} \alpha_{3}^{2}+16 \lambda_{1} \lambda_{3}+16 \lambda_{3}^{2}+6 \alpha_{1} \alpha_{3}+6 \alpha_{1}^{2}+12 \lambda_{1} y_{t}^{2}-6 y_{t}^{4}-18 \lambda_{1} g_{2}^{2}+3 g_{2}^{4}, \\
& 16 \pi^{2} \frac{\partial \lambda_{3}}{\partial t}=-\alpha_{3}^{2}+24 \lambda_{1} \lambda_{3}+16 \lambda_{3}^{2}+12 \lambda_{3} y_{t}^{2}+3 y_{t}^{4}-18 \lambda_{3} g_{2}^{2}+\frac{3}{2} g_{2}^{2}, \\
& 16 \pi^{2} \frac{\partial \alpha_{1}}{\partial t}=8 \lambda_{1} \alpha_{3}+\alpha_{3}^{2}+8 \lambda_{3} \alpha_{3}+16 \rho_{1} \alpha_{3}+8 \rho_{2} \alpha_{3}+3 \rho_{3} \alpha_{3}+20 \lambda_{1} \alpha_{1}+8 \lambda_{3} \alpha_{1}+16 \rho_{1} \alpha_{1}+8 \rho_{2} \alpha_{1}+6 \rho_{3} \alpha_{1}+4 \alpha_{1}^{2}+6 \alpha_{1} y_{t}^{2} \\
& -6 \alpha_{1} g_{B L}^{2}-21 \alpha_{1} g_{2}^{2}+6 g_{2}^{4}+\frac{\mathbf{1}}{\mathbf{1 6} \pi^{2}}\left(-\mathbf{1 8} \alpha_{1}^{3}-\mathbf{1 0} \alpha_{1}^{2} \alpha_{3}-\alpha_{1} \kappa_{1}^{2}-\mathbf{6} \alpha_{3}^{3}-\frac{\mathbf{2 7}}{\mathbf{2}} \alpha_{1} \alpha_{3}^{2}\right), \\
& 16 \pi^{2} \frac{\partial \alpha_{3}}{\partial t}=4 \lambda_{1} \alpha_{3}+4 \alpha_{3}^{2}-8 \lambda_{3} \alpha_{3}+4 \rho_{1} \alpha_{3}-8 \rho_{2} \alpha_{3}+8 \alpha_{1} \alpha_{3}+6 \alpha_{3} y_{t}^{2}-6 \alpha_{3} g_{B L}^{2}-21 \alpha_{3} g_{2}^{2} \\
& +\frac{1}{16 \pi^{2}}\left(-34 \alpha_{1}^{2} \alpha_{3}-34 \alpha_{1} \alpha_{3}^{2}-\frac{19}{2} \alpha_{3}^{3}-\alpha_{3} \kappa_{1}^{2}\right),
\end{aligned}
$$

\footnotetext{
${ }^{4}$ One can also get the total one-loop $\beta$ functions of the minimal LRSM from the website https://github.com/jlgluza/LR.
} 


$$
\begin{gathered}
16 \pi^{2} \frac{\partial \rho_{1}}{\partial t}=2 \alpha_{3}^{2}+28 \rho_{1}^{2}+16 \rho_{1} \rho_{2}+16 \rho_{2}^{2}+3 \rho_{3}^{2}+4 \alpha_{1} \alpha_{3}+4 \alpha_{1}^{2}-12 \rho_{1} g_{B L}^{2}+6 g_{B L}^{4}+12 g_{B L}^{2} g_{2}^{2}-24 \rho_{1} g_{2}^{2}+9 g_{2}^{4}+\kappa_{1}^{2} \\
16 \pi^{2} \frac{\partial \rho_{2}}{\partial t}=-\alpha_{3}^{2}+24 \rho_{1} \rho_{2}+12 \rho_{2}^{2}-12 \rho_{2} g_{B L}^{2}-24 \rho_{2} g_{2}^{2}-12 g_{2}^{2} g_{B L}^{2}+3 g_{2}^{4} \\
16 \pi^{2} \frac{\partial \rho_{3}}{\partial t}=2 \alpha_{3}^{2}+32 \rho_{1} \rho_{3}+16 \rho_{2} \rho_{3}+4 \rho_{3}^{2}+8 \alpha_{1} \alpha_{3}+8 \alpha_{1}^{2}-12 \rho_{3} g_{B L}^{2}-24 \rho_{3} g_{2}^{2}+12 g_{B L}^{4}+2 \kappa_{1}^{2},
\end{gathered}
$$

where $y_{t} \simeq\left(Y_{Q_{1}}\right)_{33}$ in the small-tan $\beta$ limit denotes the top-quark Yukawa coupling, $t=\ln \mu$, and the terms in bold denote dominant two-loop contributions. Note that since the operators $\operatorname{Tr}\left(\Phi^{\dagger} \Phi\right)\left[\operatorname{Tr}\left(\Delta_{L} \Delta_{L}^{\dagger}\right)+\operatorname{Tr}\left(\Delta_{R} \Delta_{R}^{\dagger}\right)\right]$ and $\left[\operatorname{Tr}\left(\Phi \Phi^{\dagger} \Delta_{L} \Delta_{L}^{\dagger}\right)+\right.$ $\left.\operatorname{Tr}\left(\Phi^{\dagger} \Phi \Delta_{R} \Delta_{R}^{\dagger}\right)\right]$ contain the same fields, the calculations of the $\beta$ functions for $\alpha_{1}$ and $\alpha_{3}$ are entangled. Thus we also present the two-loop result for $\alpha_{1}$, although it is not important in our numerical calculation. From these analytic expressions, one can learn that for the benchmark point in Table IV, the two-loop contribution is at most $6 \%$ of its corresponding one-loop result at the $\mathrm{TeV}$ scale, although the values of $\kappa_{1}$ and $\alpha_{3}$ are quite large.

[1] G. Aad et al. (ATLAS Collaboration), Phys. Lett. B 716, 1 (2012).

[2] S. Chatrchyan et al. (CMS Collaboration), Phys. Lett. B 716, 30 (2012).

[3] J. C. Pati and A. Salam, Phys. Rev. D 10, 275 (1974); 11, 703(E) (1975).

[4] R. N. Mohapatra and J. C. Pati, Phys. Rev. D 11, 566 (1975).

[5] R. N. Mohapatra and J. C. Pati, Phys. Rev. D 11, 2558 (1975).

[6] G. Senjanovic and R. N. Mohapatra, Phys. Rev. D 12, 1502 (1975).

[7] G. Senjanovic, Nucl. Phys. B153, 334 (1979).

[8] P. Minkowski, Phys. Lett. B 67, 421 (1977).

[9] R. N. Mohapatra and G. Senjanovic, Phys. Rev. Lett. 44, 912 (1980).

[10] J. Schechter and J. W. F. Valle, Phys. Rev. D 22, 2227 (1980).

[11] G. Lazarides, Q. Shafi, and C. Wetterich, Nucl. Phys. B181, 287 (1981).

[12] R. N. Mohapatra and G. Senjanovic, Phys. Rev. D 23, 165 (1981).

[13] J. Heeck and S. Patra, Phys. Rev. Lett. 115, 121804 (2015).

[14] C. Garcia-Cely and J. Heeck, J. Cosmol. Astropart. Phys. 03 (2016) 021.

[15] S. Patra and S. Rao, Phys. Lett. B 759, 454 (2016).

[16] A. Berlin, Phys. Rev. D 93, 055015 (2016).

[17] D. Borah, S. Patra, and S. Sahoo, Int. J. Mod. Phys. A 31, 1650097 (2016).

[18] A. Berlin, P. J. Fox, D. Hooper, and G. Mohlabeng, J. Cosmol. Astropart. Phys. 06 (2016) 016.

[19] P. S. Bhupal Dev, R. N. Mohapatra, and Y. Zhang, J. High Energy Phys. 11 (2016) 077.

[20] P. S. B. Dev, R. N. Mohapatra, and Y. Zhang, Mod. Phys. Lett. A 32, 1740007 (2017).

[21] T. Bandyopadhyay and A. Raychaudhuri, Phys. Lett. B 771, 206 (2017).

[22] M. Fukugita and T. Yanagida, Phys. Lett. B 174, 45 (1986).
[23] T. j. Li, F. Wang, and J. M. Yang, Nucl. Phys. B820, 534 (2009).

[24] C. Balazs, T. j. Li, F. Wang, and J. M. Yang, J. High Energy Phys. 09 (2009) 015.

[25] C. Balazs, T. Li, F. Wang, and J. M. Yang, J. High Energy Phys. 01 (2011) 023.

[26] D. Chang, R. N. Mohapatra, and M. K. Parida, Phys. Rev. Lett. 52, 1072 (1984).

[27] D. Chang, R. N. Mohapatra, and M. K. Parida, Phys. Rev. D 30, 1052 (1984).

[28] C. Arbelaez, M. Hirsch, M. Malinsky, and J. C. Romao, Phys. Rev. D 89, 035002 (2014).

[29] G. Bambhaniya, J. Chakrabortty, J. Gluza, T. Jeliski, and M. Kordiaczynska, Phys. Rev. D 90, 095003 (2014).

[30] A. Maiezza, M. Nemevsek, and F. Nesti, Phys. Rev. D 94, 035008 (2016).

[31] A. Maiezza, G. Senjanovic, and J. C. Vasquez, Phys. Rev. D 95, 095004 (2017).

[32] J. Chakrabortty, J. Gluza, T. Jelinski, and T. Srivastava, Phys. Lett. B 759, 361 (2016).

[33] W. Y. Keung and G. Senjanovic, Phys. Rev. Lett. 50, 1427 (1983).

[34] P. Chiappetta, A. Deliyannis, A. Fiandrino, and P. Taxil, Phys. Lett. B 308, 304 (1993).

[35] A. Maiezza, M. Nemevsek, F. Nesti, and G. Senjanovic, Phys. Rev. D 82, 055022 (2010).

[36] V. Tello, M. Nemevsek, F. Nesti, G. Senjanovic, and F. Vissani, Phys. Rev. Lett. 106, 151801 (2011).

[37] M. Nemevsek, F. Nesti, G. Senjanovic, and Y. Zhang, Phys. Rev. D 83, 115014 (2011).

[38] J. N. Esteves, J. C. Romao, M. Hirsch, W. Porod, F. Staub, and A. Vicente, J. High Energy Phys. 01 (2012) 095.

[39] S. P. Das, F. F. Deppisch, O. Kittel, and J. W. F. Valle, Phys. Rev. D 86, 055006 (2012).

[40] C. Y. Chen, P. S. B. Dev, and R. N. Mohapatra, Phys. Rev. D 88, 033014 (2013).

[41] G. Bambhaniya, J. Chakrabortty, J. Gluza, M. Kordiaczyska, and R. Szafron, J. High Energy Phys. 05 (2014) 033. 
[42] G. Bambhaniya, J. Chakrabortty, J. Gluza, T. Jelinski, and R. Szafron, Phys. Rev. D 92, 015016 (2015).

[43] A. Das, N. Nagata, and N. Okada, J. High Energy Phys. 03 (2016) 049.

[44] P. S. B. Dev, R. N. Mohapatra, and Y. Zhang, J. High Energy Phys. 05 (2016) 174.

[45] P. S. Bhupal Dev, R. N. Mohapatra, and Y. Zhang, Phys. Rev. D 95, 115001 (2017).

[46] P. S. B. Dev, R. N. Mohapatra, and Y. Zhang, Nucl. Phys. B923, 179 (2017).

[47] A. Das, P. S. B. Dev, and R. N. Mohapatra, Phys. Rev. D 97, 015018 (2018).

[48] N. G. Deshpande, J. F. Gunion, B. Kayser, and F. I. Olness, Phys. Rev. D 44, 837 (1991).

[49] M. Hirsch, H. V. Klapdor-Kleingrothaus, and O. Panella, Phys. Lett. B 374, 7 (1996).

[50] Y. Zhang, H. An, X. Ji, and R. N. Mohapatra, Nucl. Phys. B802, 247 (2008).

[51] D. Guadagnoli and R. N. Mohapatra, Phys. Lett. B 694, 386 (2011).

[52] M. Blanke, A. J. Buras, K. Gemmler, and T. Heidsieck, J. High Energy Phys. 03 (2012) 024.

[53] R. L. Awasthi, M. K. Parida, and S. Patra, J. High Energy Phys. 08 (2013) 122.

[54] J. Barry and W. Rodejohann, J. High Energy Phys. 09 (2013) 153.

[55] S. Bertolini, A. Maiezza, and F. Nesti, Phys. Rev. D 89, 095028 (2014).

[56] J. Gluza and T. Jeliski, Phys. Lett. B 748, 125 (2015).

[57] R. L. Awasthi, P. S. B. Dev, and M. Mitra, Phys. Rev. D 93, 011701 (2016).

[58] G. Bambhaniya, P. S. B. Dev, S. Goswami, and M. Mitra, J. High Energy Phys. 04 (2016) 046.

[59] J. Gluza, T. Jelinski, and R. Szafron, Phys. Rev. D 93, 113017 (2016).

[60] M. Czakon, M. Zralek, and J. Gluza, Nucl. Phys. B573, 57 (2000).

[61] J. Chakrabortty, J. Gluza, R. Sevillano, and R. Szafron, J. High Energy Phys. 07 (2012) 038.

[62] DAMPE Collaboration, Nature (London) 552, 63 (2017).

[63] J. Chang et al. (DAMPE Collaboration), Astropart. Phys. 95, 6 (2017).

[64] Q. Yuan et al., arXiv:1711.10989.

[65] Y.Z. Fan, W. C. Huang, M. Spinrath, Y. L. S. Tsai, and Q. Yuan, arXiv:1711.10995.

[66] W. Chao and Q. Yuan, arXiv:1711.11182.

[67] J. Cao, L. Feng, X. Guo, L. Shang, F. Wang, and P. Wu, arXiv:1711.11452.

[68] G. H. Duan, X. G. He, L. Wu, and J. M. Yang, arXiv: 1711.11563.

[69] W. Chao, H. K. Guo, H. L. Li, and J. Shu, arXiv: 1712.00037.

[70] R. Zhu and Y. Zhang, arXiv:1712.01143.

[71] J. Cao, L. Feng, X. Guo, L. Shang, F. Wang, P. Wu, and L. Zu, Eur. Phys. J. C 78, 198 (2018).

[72] P. H. Gu and X. G. He, Phys. Lett. B 778, 292 (2018).

[73] G. H. Duan, L. Feng, F. Wang, L. Wu, J. M. Yang, and R. Zheng, J. High Energy Phys. 02 (2018) 107.

[74] L. Zu, C. Zhang, L. Feng, Q. Yuan, and Y. Z. Fan, arXiv: 1711.11052.
[75] Y. L. Tang, L. Wu, M. Zhang, and R. Zheng, arXiv: 1711.11058.

[76] P. H. Gu, arXiv:1711.11333.

[77] P. Athron, C. Balazs, A. Fowlie, and Y. Zhang, J. High Energy Phys. 02 (2018) 121.

[78] X. Liu and Z. Liu, arXiv:1711.11579.

[79] X. J. Huang, Y. L. Wu, W. H. Zhang, and Y. F. Zhou, arXiv:1712.00005.

[80] Y. Gao and Y.Z. Ma, arXiv:1712.00370.

[81] J. S. Niu, T. Li, R. Ding, B. Zhu, H. F. Xue, and Y. Wang, arXiv:1712.00372.

[82] P. H. Gu, arXiv:1712.00922.

[83] T. Nomura and H. Okada, arXiv:1712.00941.

[84] T. Li, N. Okada, and Q. Shafi, Phys. Lett. B 779, 130 (2018).

[85] C. H. Chen, C. W. Chiang, and T. Nomura, Phys. Rev. D 97, 061302 (2018).

[86] R. Ding, Z. L. Han, L. Feng, and B. Zhu, arXiv: 1712.02021.

[87] G. L. Liu, F. Wang, W. Wang, and J. M. Yang, Chin. Phys. C 42, 035101 (2018).

[88] Y. Zhao, K. Fang, M. Su, and M. C. Miller, arXiv: 1712.03210 .

[89] Y. Sui and Y. Zhang, arXiv:1712.03642.

[90] N. Okada and O. Seto, arXiv:1712.03652.

[91] K. Ghorbani and P. H. Ghorbani, arXiv:1712.01239.

[92] H. B. Jin, B. Yue, X. Zhang, and X. Chen, arXiv: 1712.00362.

[93] I. Cholis, T. Karwal, and M. Kamionkowski, arXiv: 1712.00011.

[94] K. Fang, X. J. Bi, and P. F. Yin, Astrophys. J. 854, 57 (2018).

[95] F. Yang and M. Su, arXiv:1712.01724.

[96] S. F. Ge and H. J. He, arXiv:1712.02744.

[97] S. Profumo, F. S. Queiroz, J. Silk, and C. Siqueira, J. Cosmol. Astropart. Phys. 03 (2018) 010.

[98] C. Bonilla, M. E. Krauss, T. Opferkuch, and W. Porod, J. High Energy Phys. 03 (2017) 027.

[99] R. Slansky, Phys. Rep. 79, 1 (1981).

[100] Y. Kawamura, Prog. Theor. Phys. 103, 613 (2000).

[101] L. J. Hall and Y. Nomura, Phys. Rev. D 64, 055003 (2001).

[102] F. Staub, Adv. High Energy Phys. 2015, 840780 (2015).

[103] W. Porod, Comput. Phys. Commun. 153, 275 (2003).

[104] W. Porod and F. Staub, Comput. Phys. Commun. 183, 2458 (2012).

[105] P. A. R. Ade et al. (Planck Collaboration), Astron. Astrophys. 571, A16 (2014).

[106] J. Dunkley et al. (WMAP Collaboration), Astrophys. J. Suppl. Ser. 180, 306 (2009).

[107] G. Blanger, F. Boudjema, A. Pukhov, and A. Semenov, Comput. Phys. Commun. 192, 322 (2015).

[108] G. Belanger, F. Boudjema, and A. Pukhov, arXiv: 1402.0787.

[109] K. Griest and D. Seckel, Phys. Rev. D 43, 3191 (1991).

[110] E. Aprile et al. (XENON Collaboration), Phys. Rev. Lett. 119, 181301 (2017).

[111] X. Cui et al. (PandaX-II Collaboration), Phys. Rev. Lett. 119, 181302 (2017).

[112] A. Berlin, D. Hooper, and S. D. McDermott, Phys. Rev. D 89, 115022 (2014). 
[113] H. Abdallah et al. (H.E.S.S. Collaboration), Phys. Rev. Lett. 117, 111301 (2016).

[114] M. Ackermann et al. (Fermi-LAT Collaboration), Phys. Rev. Lett. 115, 231301 (2015).

[115] T. R. Slatyer, Phys. Rev. D 93, 023527 (2016).

[116] T. R. Slatyer, Phys. Rev. D 93, 023521 (2016).

[117] M. G. Aartsen et al. (IceCube Collaboration), Eur. Phys. J. C 77, 627 (2017).

[118] R. Essig, T. Volansky, and T. T. Yu, Phys. Rev. D 96, 043017 (2017).

[119] P. Bechtle, S. Heinemeyer, O. Stal, T. Stefaniak, and G. Weiglein, Eur. Phys. J. C 74, 2711 (2014).

[120] P. Bechtle, O. Brein, S. Heinemeyer, G. Weiglein, and K. E. Williams, Comput. Phys. Commun. 181, 138 (2010).

[121] P. Bechtle, O. Brein, S. Heinemeyer, G. Weiglein, and K. E. Williams, Comput. Phys. Commun. 182, 2605 (2011).

[122] P. Bechtle, O. Brein, S. Heinemeyer, O. Stal, T. Stefaniak, G. Weiglein, and K. E. Williams, Eur. Phys. J. C 74, 2693 (2014).

[123] W. Porod, F. Staub, and A. Vicente, Eur. Phys. J. C 74, 2992 (2014).

[124] M. E. Peskin and T. Takeuchi, Phys. Rev. D 46, 381 (1992).
[125] L. Lavoura and L. F. Li, Phys. Rev. D 49, 1409 (1994).

[126] M. Baak, J. Cúth, J. Haller, A. Hoecker, R. Kogler, K. Mönig, M. Schott, and J. Stelzer (Gfitter Group), Eur. Phys. J. C 74, 3046 (2014).

[127] C. Patrignani et al. (Particle Data Group), Chin. Phys. C 40, 100001 (2016).

[128] J. Chakrabortty, P. Konar, and T. Mondal, Phys. Rev. D 89, 056014 (2014).

[129] J. Chakrabortty, P. Konar, and T. Mondal, Phys. Rev. D 89, 095008 (2014).

[130] B. W. Lee, C. Quigg, and H. B. Thacker, Phys. Rev. D 16, 1519 (1977).

[131] A. G. Akeroyd, A. Arhrib, and E. M. Naimi, Phys. Lett. B 490, 119 (2000).

[132] J. Cao, P. Wan, J. M. Yang, and J. Zhu, J. High Energy Phys. 08 (2013) 009.

[133] F. Lyonnet, I. Schienbein, F. Staub, and A. Wingerter, Comput. Phys. Commun. 185, 1130 (2014).

[134] F. Lyonnet, arXiv:1510.08841.

[135] F. Lyonnet and I. Schienbein, Comput. Phys. Commun. 213, 181 (2017).

[136] I. Z. Rothstein, Nucl. Phys. B358, 181 (1991). 